\title{
La théorie de la vision chez Galien : la colonne qui saute et autres énigmes
}

Heinrich Von Staden

\section{(2) OpenEdition}

12 Journals

Édition électronique

URL : https://journals.openedition.org/philosant/936

DOI : 10.4000/philosant.936

ISSN : 2648-2789

Éditeur

Éditions Vrin

\section{Édition imprimée}

Date de publication : 1 novembre 2012

Pagination : 115-155

ISBN : 978-2-7574-0400-3

ISSN : 1634-4561

\section{Référence électronique}

Heinrich Von Staden, "La théorie de la vision chez Galien : la colonne qui saute et autres énigmes », Philosophie antique [En ligne], 12 | 2012, mis en ligne le 01 novembre 2018, consulté le 03 décembre 2022. URL : http://journals.openedition.org/philosant/936 ; DOI : https://doi.org/10.4000/philosant. 936

\section{(c) (i) () $\Theta$}

Creative Commons - Attribution - Pas d'Utilisation Commerciale - Pas de Modification 4.0 International - CC BY-NC-ND 4.0

https://creativecommons.org/licenses/by-nc-nd/4.0/ 


\section{LA THÉORIE DE LA VISION CHEZ GALIEN : LA COLONNE QUI SAUTE ET AUTRES ÉNIGMES*}

Heinrich VON STADEN

Institute for Advanced Study, Princeton

RÉSUMÉ. Du point de vue méthodologique et épistémologique, la vision occupe une place privilégiée dans les œuvres de Galien de Pergame, ce qui explique les tentatives répétées de ce dernier pour en expliquer le fonctionnement. En partie grâce à la dissection et à la vivisection pratiquées sur des animaux de différentes espèces, il développa une connaissance détaillée de l'anatomie de l'œil, du nerf optique, du cerveau, des muscles oculaires et du système vasculaire cérébral et oculaire. Il utilisa avec habileté cet impressionnant savoir anatomique pour élaborer une explication détaillée de la physiologie de la vision. Pour expliquer comment voient les êtres humains, il fit également appel à l'optique mathématique, notamment à l'optique euclidienne. Cependant, en dépit d'efforts remarquables pour intégrer anatomie, physiologie et mathématiques dans une unique théorie cohérente de la vision, une analyse plus attentive montre que son explication de la vision n'est pas exempte de lacunes non négligeables, de difficultés non résolues et de tensions internes.

SUMMARY. Vision enjoys a privileged methodological and epistemological position in the works of Galen of Pergamon, and he therefore made repeated attempts to explain how vision works. In part through his dissections and vivisections of a variety of nonhuman animals, he developed a detailed knowledge of the anatomy of the eye, of the optic nerve, of the brain, of the oculomotor muscles, and of the cerebral and ocular arteries and veins. He deftly used this impressive anatomical knowledge to construct an elaborate physiological account of vision. In addition, he drew on mathematical optics, notably on Euclidian optics, in order to explain how and what human beings see. Despite his remarkable efforts to unite anatomical, physiological and mathematical strands within a single coherent theory of vision, however, a closer analysis shows that his account is not free of significant explanatory lacunae, unresolved puzzles, and internal tensions.

: Cette contribution a son origine dans une conférence présentée au Centre Léon Robin. Je suis très reconnaissant aux participants à cette occasion, et notamment à Pierre Pellegrin et à Gilbert Romeyer Dherbey, pour leurs fort utiles commentaires. Je remercie aussi les directeurs de Philosophie antique pour leur aide et leur disponibilité. 



\section{Introduction}

La valorisation épistémologique et méthodologique de la vision par Galien l'a amené à examiner la nature de la vision humaine dans presque chaque période de sa prolifique activité littéraire ${ }^{1}$. Il accorde à la vision une position privilégiée parmi les cinq sens, en affirmant qu'elle est supérieure aux autres sens en puissance (dynamis) et en précision (akribeia). De plus, il décrit fréquemment l'observation visuelle à la fois comme un point de départ heuristique et comme un outil vérificateur central, même lorsqu'il présente sa science du corps comme une science axiomatique, susceptible d'être parachevée en procédant par démonstration à partir de données indémontrables, à savoir des propositions de base ou archai qui sont autojustificatrices, fondations métaphysiquement antérieures ${ }^{2}$. La vision, en d'autres termes, ne se contente pas de fournir à la partie raisonnante de l'âme, que Galien, suivant Platon et d'autres penseurs, place dans le cerveau $^{3}$, les témoignages indispensables; elle est aussi un moyen grâce

1. Les témoignages nous lancent un défi redoutable, car au moins quatre des traités dans lesquels Galien a exposé sa théorie ont été perdus : De la démonstration, livres V et XIII ; Des couleurs; Commentaires sur le Timée de Platon (dont ne nous sont parvenus que des fragments); Diagnostic des affections des yeux (voir n. 60). En outre, parmi ceux qui nous sont parvenus, les plus significatifs de ses exposés sur la vision sont éparpillés à travers sept traités : De symptomatum causis, De locis affectis, De symptomatum differentiis, De anatomicis administrationibus, De nervorum dissectione, De usu partium (dorénavant UP), De placitis Hippocratis et Platonis (dorénavant $P H P$ ). De plus, pour plusieurs de ces textes, il n'existe aucune édition critique, et là où les détails éparpillés de la théorie de la vision de Galien ne sont pas obscurs, ils semblent fréquemment lacunaires ou contradictoires. Galien fait aussi plus d'une fois référence à ses optikoi logoi, mais il n'est pas sûr qu'il s'agisse d'un texte à part et indépendant ; cf. UP 8.6, 10.6 (III, p. 641, 785-786 Kühn [dorénavant K.] = I, p. 464 et II, p. 74 Helmreich 1907 [dorénavant Helmr.]) ; In Hippocratis Epidemiarum 6.4.22 comm. 4.29 (XVII B, p. 214 K. = CMG V 10, 2, 2, p. 247 Wenkebach). De même, dans le De

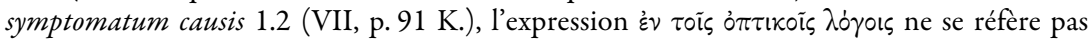
forcément à un traité à part, mais peut-être aux exposés de la vision dans $U P$ et dans $P H P$. Pour l'analyse de la vision dans son traité De la démonstration, voir p. ex. UP 16.3 (IV, p. 275 K. = II, p. 384 Helmr.) ; PHP 7.4.4, 7.5.36-37, 7.5.39, 7.6.24 (V, p. 612, 626, 634 K. = CMG V 4, 1, 2, p. 448, 460, 466 De Lacy); In Hippocratis Prognosticum comm. 1, 23 (XVIII B, p. 72-73 K. = CMG V 9, 2, p. 236 Heeg); cf. von Müller 1895; Jaeger 1914, p. 17,36; Chiaradonna 2009; Havrda 2011. Voir infra n. 69.

2. Sur la théorie de la méthode scientifique de Galien et sur son épistémologie, voir von Müller 1895; Frede 1981 (repris dans Frede 1987, p. 279-298); Vegetti 1981; Barnes 1982 ; Barnes 1991 ; Hankinson 1991a ; Barnes 1993 ; Vegetti 1994, notamment p. 17081717; Barnes 2003; Hankinson 2008a; Morison 2008; Tieleman 2008; Chiaradonna 2011.

3. P. ex. $P H P$ 2.3.24, 5.7.1-4, 8.1.22 (V, p. 225, 479-480, 655 K. = CMG V, 4,1,2, p. 114, 336, 484-486 De Lacy); De methodo medendi, 13.21 (X, p. 929 K). Cf. PHP 2.4.17 
auquel des hypothèses peuvent être vérifiées, et une pierre de touche pour éprouver ou confirmer les résultats d'une démonstration.

Pour établir la supériorité cognitive de la vision, Galien tisse un réseau théorique complexe de fils anatomiques, physiologiques et mathématiques qui se soutiennent mutuellement, nombre d'entre eux étant intégrés dans une perspective téléologique englobante. Réunis, ces fils conduisent aux réponses, problématiques mais extrêmement influentes ${ }^{4}$, de Galien à des questions telles que : en quoi consiste la transmutation du visible en vu ? Comment voyons-nous? Que voyons-nous ? Et pourquoi la vue est-elle le sens supérieur? La question peut-être la plus fondamentale, et en même temps la plus difficile, entraînée par les nombreuses discussions de Galien sur la vision est de savoir si ces fils forment un tissu d'une pièce, cohérent, ou s'ils se superposent de façon telle qu'ils produisent des tensions, discordances ou incompatibilités conceptuelles et théoriques ${ }^{5}$. Une approche

(V, p. $230 \mathrm{~K} .=$ p. 120 De Lacy). Alors que Galien, pour certains aspects de sa théorie de l'âme, s'est montré inconstant, il a invariablement identifié le cerveau comme la source principale (arche) et le siège de la perception et de la raison. Pour sa théorie de l'âme, voir Manuli et Vegetti (éd.) 1988; Hankinson 1991b, notamment p. 208-217; Hankinson 1991c; Hankinson 1993; Tieleman 1996; Tieleman 2003; Hankinson 2006; Donini 2008.

4. Pour des exemples de l'influence de la théorie de la vision de Galien, voir Lindberg 1967 ; Lindberg 1976, p. 2, 31, 33, 38, 40, 53, 57, 67, 68; Lindberg 1978, notamment p. 143-154; Sabra 1978, surtout p. 164-165; Sabra 1989, vol. 2, p. xxxiv n. 32, lxiii n. 93, 18, 46-47, 50-51, 53, 60, 105, 140 ; Eastwood 1980 et 1982. Cf. Pines 1955.

5. Au XXe siècle les débats sur la théorie de la vision de Galien ont eu tendance à se concentrer sur des questions de provenance et d'influence plutôt que sur la cohérence systématique de la théorie. Werner Jaeger et Karl Reinhardt, par exemple, s'intéressaient surtout à ce qu'ils pensaient être l'origine posidonienne de la théorie de la vision de Galien ; Harold Cherniss à son tour a affirmé que Galien avait emprunté sa théorie principalement au Timée de Platon; David Lindberg, par contraste, s'est concentré sur des éléments tirés du stoïcisme et d'Euclide dans la théorie galénique, tandis que Paul Moraux a affirmé que Galien, comme son contemporain Alexandre d'Aphrodise, dépendait d'un compte-rendu critique - maintenant perdu - de la théorie de la vision d'Aristote (compte-rendu qui, selon Moraux, mettait en évidence des contradictions au sein des explications aristotéliciennes de la vision dans le De Anima et les Meteorologica); voir Jaeger 1914, p. 27-53; Reinhardt 1926, p. 188-192; Cherniss 1933 (=Cherniss 1977, p. 447-454), p. ex. 1933, p. 161 : «Galen's theory is simply that of Plato influenced in one detail by Aristotle 》 (je souligne; l'analyse de Cherniss se fonde sur des notes, jamais publiées, de Roger M. Jones) ; Lindberg 1976, p. 10 ( «The basic features of the Stoic theory of vision were adopted and elaborated by Galen ») et p. 12 ( 《Galen then proceeds to a brief summary of what is evidently Euclidean optics »); Moraux 1984, p. 757-762; van der Eijk 2010. Voir aussi Siegel 1970a et 1970b, p. 10-126 (contributions utiles mais entachées d'erreurs de traduction et parfois de contresens sur le grec de Galien). La dynamique du raisonnement de Galien est sans aucun doute co-déterminée par un rapport complexe, et en partie profondément agonistique, avec ses devanciers philosophes, médecins et mathématiciens, mais il faut également mettre en 
utile pour y répondre est peut-être d'examiner d'abord quelques-uns de ces fils individuellement. Comme chez Galien, l'analyse commencera par l'anatomie (II), pour passer ensuite à la physiologie (III) et à la géométrie (IV) de la vision.

\section{L'anatomie de la vision: cerveau, nerf et œil}

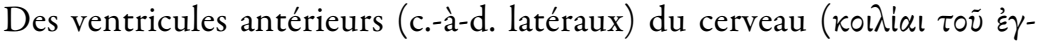
$\kappa \varepsilon \phi \dot{\alpha} \lambda \circ v)$, deux ramifications naturelles ( $\dot{\alpha} \pi \circ \phi \dot{v} \sigma \varepsilon ા \varsigma)$ partent vers l'œil, selon Galien ${ }^{6}$. Chacune de ces ramifications possède deux tuniques; l'interne, la plus molle (figure a, 1) est une excroissance de la membrane fine (pie-mère) qui enveloppe le cerveau, alors que l'externe, plus dure (fig. a, 2) est une excroissance de la membrane épaisse (dure-mère) qui enveloppe le cerveau ${ }^{7}$. À l'intérieur de cette double protection se trouve le nerf optique (fig. a, 3) qui, nous dit Galien, est fort mou $^{8}$. Par ces aspects-là, le nerf optique ne diffère pas des autres nerfs sensoriels : tous ont en commun une structure de protection double ainsi qu'une consistance molle et une source cérébrale (alors que les nerfs moteurs sont plus durs et, d'après Galien, prennent leur source dans la moelle épinière) $)^{9}$.

lumière le degré de cohérence systématique de la théorie galénique ainsi que les tensions au sein de sa théorie - une approche prometteuse, comme l'ont montré des contributions plus récentes, telles que Boudon 2002 et Lehoux 2007.

6. L'original grec des livres IX, 6-14 et X (en entier) du De anatomicis administrationibus de Galien - livres dans lesquels il traite du cerveau, des nerfs, et de l'œil - a été perdu, mais survit dans une traduction arabe du IX $\mathrm{X}^{\mathrm{c}}$ siècle, faite par Hubaish, le neveu de Hunain ibn Ishaq. Hubaish a fondé sa traduction sur une traduction syriaque faite par Ayyub d'Edessa, révisée par la suite (ca. 850) par Hunain à la lumière de trois manuscrits grecs maintenant perdus. Hunain, semble-t-il, a aussi révisé la traduction arabe d'Hubaish, qui, sous sa forme révisée, subsiste dans plusieurs manuscrits. Mes citations de cette version arabe s'appuient sur l'édition du texte arabe par Simon 1906, t. I (dorénavant Simon), et sur trois traductions modernes: Simon 1906, t. II ; Duckworth, Lyons, Towers 1962 (dorénavant D.) ; Garofalo 1991a, t. 2, p. 827-854 et t. 3 (dorénavant G.). Sur les branches ou ramifications partant des ventricules cérébraux, voir Anat. adm. 9.9, 9.10, 14.2, 14.4 (p. 9, 18, 235-236, 246 Simon = p. 8, 14, 187-188, 195 D. = p. 834, 842, 1044-1045, 1053-1054 G.).

7. Anat. adm. 10.3 (p. 46 Simon = p. 38 D. = p. 869 G.).

8. Galien compare la substance matérielle du nerf optique au lait qui commence tout juste à cailler, alors que la substance des autres nerfs sensoriels ressemble à celle du lait déjà caillé, c'est-à-dire qu'ils sont plus denses et plus compacts: Anat. adm. 14, 2 (p. 235, 237 Simon = p. 187, 188 D. = p. 1044, 1045-1046 G.). Voir aussi infra n. 21 pour l'utilisation d'analogies similaires pour décrire l'humeur vitrée et le cristallin.

9. Anat. adm. 7.8 (II, p. 612-613 K. = p. 652-654 G.) ; UP 8.5-6, 9.8, 9.11, 9.13-14 (III, p. 633-639, 717-718, 724-725, 739-743 K. = I, p. 458-463; II, p. 25-26, 30-31, 41-44 Helmr.) ; PHP 7.5.16 (V, p. 622 K. = p. 456 De Lacy). Voir infra n. 48. 


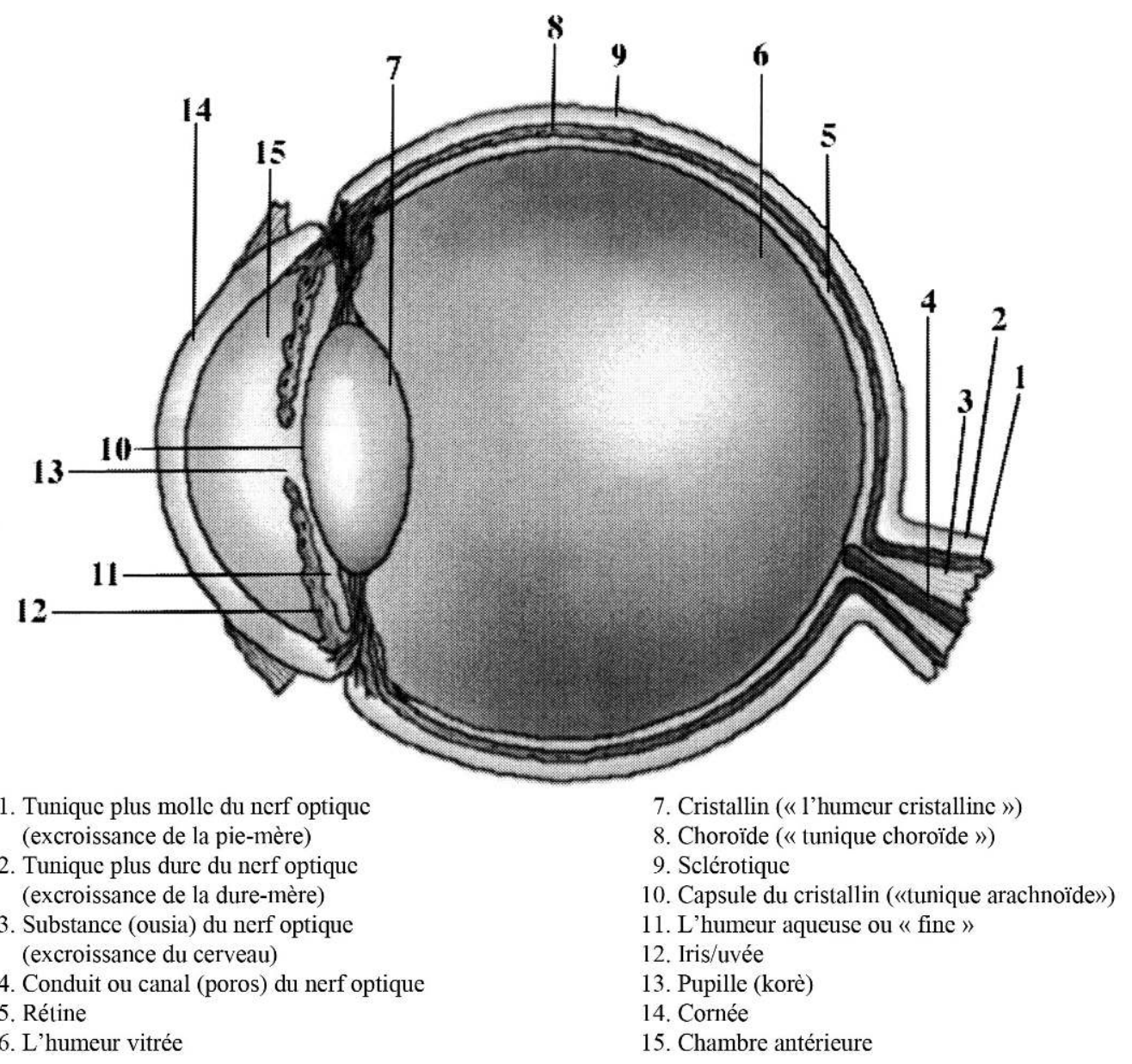

Fig. a

L'œil selon Galien

Le nerf optique possède pourtant des traits anatomiques qui le distinguent des autres nerfs sensoriels et aident ainsi à rendre compte, anatomiquement, de ses pouvoirs uniques, selon Galien. Cinq de ces traits distinctifs valent la peine d'être notés. Tout d'abord, c'est le plus large de tous les nerfs en diamètre, le seul qui comporte un conduit intérieur per- 
ceptible (poros, «passage », « canal »), qui court sur toute sa longueur au centre du nerf ( $c f$. fig. a, 4$)^{10}$. En deuxième lieu, c'est le plus mou de tous les nerfs ${ }^{11}$. Troisièmement, alors que tous les nerfs sensoriels s'avancent par paires hors du cerveau, les nerfs optiques sont la seule paire dont les éléments se joignent et se séparent au long de leur course vers leur organe sensoriel $^{12}$. Avant de sortir du crâne (kranion) par un petit orifice (trema, le foramen optique) pour entrer dans l'espace orbitaire de chaque œil, les deux nerfs optiques se joignent, comme Galien l'a justement observé, pour former un « chi » $(\mathrm{X})$, c.-à-d. le chiasma optique. Cependant, d'après Galien, aucun des deux nerfs ne passe effectivement de l'autre côté ; ou plutôt, ils s'unissent et se séparent en sorte que le nerf optique qui trouve son origine dans la partie inférieure droite du ventricule antérieur (latéral) droit du cerveau pénètre finalement dans l'œil droit, et vice versa ${ }^{13}$. Galien souligne qu'aucune autre paire de nerfs ne forme un tel chiasma. Quatrièmement, quand le nerf optique pénètre dans la partie arrière ou « racine » ( $\dot{i} i \zeta)$ de l'œil, nous dit Galien, il devient « relâché » ou « détendu » et aplati et il s'étend, « semblable à un filet de pêche » $\left(\dot{\alpha} \mu \phi ı \beta \lambda \eta \sigma \tau \rho \circ \varepsilon \delta_{\eta} \varsigma^{14}\right)$, c'est-à-dire qu'il devient la rétine (fig. a, 5) ${ }^{15}$. Aucun autre nerf ne se comporte ainsi. Cinquièmement, Galien affirme à plusieurs reprises, non seulement que la

10. Anat. adm. 9.8, 10.2, 14.2 (p. 7, 46, 238 Simon = p. 6, 38, 188 D. $=$ p. 833,869 , 1046 G.). Sur la taille du nerf optique et son conduit, voir aussi Anat. adm. 9.9, 10.1, (p. 9 , 34-35 Simon = p. 8, 29 D. = p. 834-835 G.); mais ibid. 14.1 (p. 235 Simon = p. 187 D. = p. 1044 G.), Galien souligne que le canal creux du nerf optique (bien que plus large que celui de tout autre nerf) est relativement étroit et difficile à observer: « ... il s'agit seulement d'un petit passage creux, difficile à exposer à la vue. On ne peut le détecter que par l'insertion d'une sonde fine, une soie de sanglier ou quelque autre objet d'une finesse comparable ». Voir aussi $U P$ 8.6, 10.12, 16.3 (III, p. 639, 641-642, 644, 813, IV, p. $275 \mathrm{~K} .=\mathrm{I}$, p. 463, 465, 467 ; II, p. 93, 384 Helmr.) ; PHP 7.4.4-10, 7.5.17 (V, p. 612-614, $622 \mathrm{~K}$. $=$ p. 448-450, 456 De Lacy); De nervorum dissectione, 2 (II, p. 832-833 K. = p. 27-28

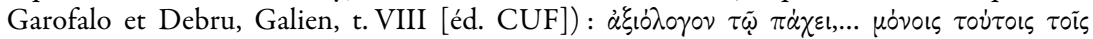

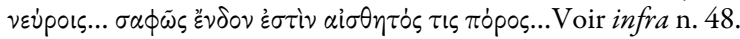

11. UP 16.3 (IV, p. 273 K. = II, p. 382-383 Helmr.) ; De nervorum dissectione, 2 (II, p. $832-833$ K.) : $\mu \alpha \lambda \alpha \kappa \dot{\omega} \tau \alpha \tau \circ \nu, \mu \alpha \lambda \alpha \kappa \omega \tau \dot{\alpha} \tau \eta \nu$.

12. Anat. adm. 9.8, 10.1, 14.2 (p. 7, 33, 236 Simon = p. 6, 28, 187-188 D. = p. 832-833, 857-858, 1045 G.); UP 9.8, 10.12, 10. 13-14 (III, p. 712-713, 813-814, 829-837 K. = II, p. 22, 93-94, 104-110 Helmr.). Voir infra n. 96-97.

13. Anat. adm. 14.2 (p. 236 Simon = p. 187-188 D. = p. 1045 G.) ; UP 10.12 (III, p. 813-814 K. = II, p. 93-94 Helmr.).

14. Le mot latin médiéval retina (dérivé de rete) est un calque du terme grec.

15. Anat. adm. 10.2-3, 14.2 (p. 46-47, 237 Simon = p. 38-39, 188 D. = p. 869-870, 1045-1046 G.) ; UP 8.6, 10.1-2, 10.13 (III, p. 639, 642-643, 760, 761-768, $830 \mathrm{~K} .=\mathrm{I}$, p. 463, 466 ; II, p. 55-61, 105 Helmr.) ; PHP 7.5.25-28 (V, p. 624 K. = p. 458 De Lacy); De nervorum dissectione, 2 (II, p. 832-833 K. = p. 27-28 Garofalo et Debru). Voir aussi Meth. med. 1.6 (X, p. $47 \mathrm{~K})$. 
rétine elle-même est nerf, mais encore que si on l'ôtait soigneusement du reste de l'œil et qu'on la repliait sur elle-même, on ne pourrait pas la distinguer de la véritable substance du cerveau ${ }^{16}$ : « Après être entré dans l'œil, le nerf optique est libéré de son confinement et devient entièrement, sous tous ses aspects, semblable au cerveau ( $\dot{\xi} \xi 0 \mu 010 \tilde{\sigma} \sigma \theta \alpha l \quad k \alpha \tau \dot{\alpha} \quad \pi \dot{\alpha} \nu \tau \alpha \quad \dot{\varepsilon} \gamma-$ $\kappa \varepsilon \phi \dot{\alpha} \lambda \omega)^{17}$. » Cela aussi est un trait distinctif de la relation du nerf optique à son organe, l'œil : « On ne trouvera la substance-même qui se trouve dans le cerveau dans aucun autre organe (des sens) ${ }^{18}$, c.-à-d. mis à part l'œil. La rétine est donc à la fois nerf sensoriel et substance cérébrale.

Dans la construction galénique d'une anatomie fonctionnelle, menée téléologiquement, l'unicité de structure anatomique tend à être en corrélation avec l'unicité d'utilité (chreia), d'activité (energeia), de pouvoir ( $\delta \dot{v} v \alpha-$ $\mu l \varsigma$ : la capacité d'avoir ou de subir un effet) et de fonction ("̈pyov) - corrélation que Galien tourne à l'avantage de sa valorisation épistémologique de la vision, comme nous allons le voir (III).

Indispensable à la compréhension de la théorie galénique de la vision est son anatomie du globe oculaire (fig. $\mathrm{a}^{19}$ ) qui est une élaboration de l'anatomie remarquablement précise de l'œil d'Hérophile ${ }^{20}$. Galien souligne non seulement l'unicité du nerf optique mais encore le caractère unique de la structure et de la composition matérielle de l'œil. Elle consiste, grosso modo, en deux corps sphériques humides - aucun des deux n'est une sphère parfaite - et en une série d'enveloppes partielles, radicalement différentes entre elles, qui les recouvrent. Le corps sphérique postérieur, le plus mou, a la consistance, la transparence et la couleur du verre partiellement fondu et

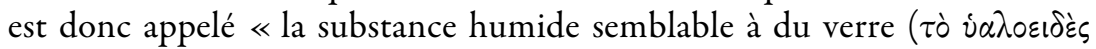
ípóv)» (fig. a, 6), qui, par la médiation de sa traduction latine est devenue connue sous le nom $d$ '《 humeur vitrée »), alors que la sphère antérieure, plus dure et beaucoup plus petite, est appelée « la substance humide sem-

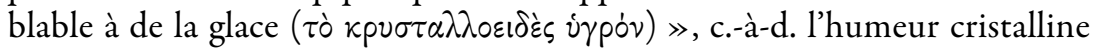

16. UP 10.2 (III, p. 762 K. = II, p. 56 Helmr.); PHP 7.5.22, 7.5.28 (V, p. 623, $624 \mathrm{~K}$. = p. 458 De Lacy). Voir aussi UP 8.6 (III, p. $641 \mathrm{~K} .=$ I, p. 465 Helmr.) : le cerveau étend « une partie de lui-même» (c.-à-d. le nerf optique et la rétine) jusqu'au cristallin; $D e$ nervorum dissectione, 2 (II, p. 833, 4-5 K. = p. 28, 3 Garofalo et Debru) : chacun des deux nerfs optiques pénètre dans l'œil «sans s'écarter en rien à cet endroit de la nature du

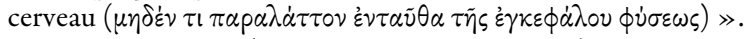

17. PHP 7.5.17 (V, p. $622 \mathrm{~K} .=456$ De Lacy).

18. PHP 7.5.30 (V, p. $625 \mathrm{~K} .=458$ De Lacy).

19. Comme la plupart des représentations schématiques de phénomènes complexes, la figure a est, bien sûr, réductrice et lacunaire, mais elle donne une idée de la version anatomique de l'œil de Galien.

20. Voir von Staden 1989, p. 160-161, 202-206, 237-239, 252-254, 317-318 (T140a); id. 2002. 
ou le cristallin (fig. a, 7) ${ }^{21}$. Chacun de ces deux corps sphériques est, d'après Galien, « parfaitement clair, transparent et brillant » $(\kappa \alpha \theta \alpha \rho \dot{\alpha} \kappa \alpha \grave{\iota} \delta ı \alpha v \gamma \tilde{\eta}$ $\kappa \alpha i \lambda \alpha \mu \pi \rho \dot{\alpha})^{22}$.

Le corps sphérique postérieur dans l'œil (l'humeur vitrée) possède trois enveloppes partielles d'une dureté croissante de l'intérieur vers l'extérieur, chacune émanant du nerf optique. Directement étalée sur l'humeur vitrée se trouve l'extension directe, très molle, aplatie, du nerf optique : la rétine,

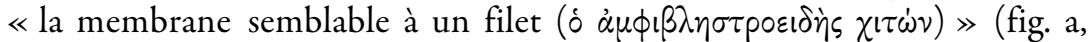

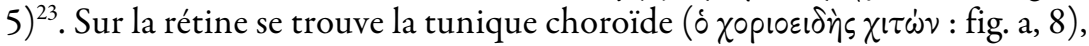
un peu plus dure, continuation directe de l'enveloppe interne du nerf optique, c.-à-d. de la couche qui est une excroissance de la pie-mère ${ }^{24}$. Sur la tunique choroïde est étalée à son tour une membrane encore plus dure, la

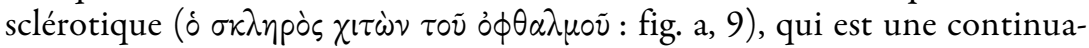
tion directe de l'enveloppe extérieure du nerf optique, c.-à-d. une excroissance de la dure-mère ${ }^{25}$. Aucune de ces trois membranes ne s'étend tout autour de l'humeur vitrée, comme l'illustre la figure a : sur ses faces postérieure et antérieure, l'humeur vitrée est structurée de façon à laisser des

21. Sur l'humeur vitrée et le cristallin voir Anat. adm. 10.3 (p. 47-53 Simon = 39-43 D. $=870-875$ G.). Du cristallin (littéralement, « ce qui est semblable à de la glace »), Galien dit cependant : « Ce nom lui a été donné parce que son aspect ressemble à celui de la glace; mais si on le manie, on sent qu'il est beaucoup moins dur que de la glace $\gg$ (Anat. adm. 10.2 [p. 42-43 Simon = 35-36 D. = 866 G.]). Voir aussi UP 8.5, 8.6, 10.1-4, 10.6, 10.13 (III, p. 635, 644, 760-762, 766-769, 777-781, 786-789, 830-836; IV, p. $160 \mathrm{~K} .=\mathrm{I}$, p. 460, 467 ; II, p. 55-57, 59-61, 68-71, 75-76, 105 Helmr.) ; PHP 5.3.6, 7.5.23-26, 9.8 .17 (V, p. 446, 623-624, 788 K. = 306, 458, 594 De Lacy). Cf. Meth. med. 1.6, 2.6, 7.2 (X, p. 48-49, 118119, 459-460 K.) ; De bonis malisque sucis, 6.6 (VI, p. 789 K. = CMG V 4, 2, p. 412 Helmr.) ; De symptomatum causis, 1.2-3 (VII, p. 86-87, 89-91, 93-94, 96-97, 99, 103 K.). Galien compare aussi l'humeur du cristallin à du fromage humide ou liquide et la substance du corps vitré à un fromage encore plus humide et liquide, comme solidifié à demi seulement; Anat. adm. 10.3 (p. 49 Simon $=40$ D. $=872$ G.). Cf. supra n. 8.

22. PHP 7.5.24 (V, p. $623 \mathrm{~K} .=458$ De Lacy). Cf. UP 8.6, 10.1 (III, p. 641, $761 \mathrm{~K} .=\mathrm{I}$, p. 465; II, p. 55-56 Helmr.).

23. Voir supra n. 15-18.

24. UP 10.2-3 (III, p. 762-768, 771, 778 K. = II, p. 57-61, 63, 68 Helmr.). Dans la traduction arabe de l'original grec perdu du dixième livre du De anatomicis administrationibus, la membrane choroïde de l'œil est appelée «la seconde tunique » ou «la seconde couche » (c.-à-d. entre la rétine et la sclérotique); Anat. adm. 10. 2-3 (p. 45-47 Simon = 3839 D. $=868-870$ G. $)$.

25. Sur la sclérotique, voir Anat. adm. 10.2 (p. 40,43-44, 46 Simon = p. 33-34, 36-37, 38 D. $=$ p. $864,867,869$ G.). Dans ces passages, Galien décrit la sclérotique comme, entre autres, une tunique ou membrane dure, croquante, nommée cartilagineuse par certains, qui est perforée là où le nerf optique entre dans le côté postérieur de l'œil, et qui s'étend autour de l'humeur vitrée jusqu'à la couronne ou jonction de la sclérotique et de la cornée, d'où la sclérotique devient une tunique stratifiée à quatre couches, à savoir la cornée (voir infra n. 33). Cf. UP 10.2-3 (III, p. 764, 767-768, 770-771 K. = II, p. 58, 60-61, 63 Helmr.). 
moyens de communication essentiels avec, respectivement, le cerveau et le cristallin. Sur sa face postérieure se trouve une ouverture par laquelle le pneuma psychique sensoriel, transmis des ventricules antérieurs du cerveau par le large conduit (poros: fig. a, 4) dans le nerf optique, peut entrer dans l'œil, et sur sa face antérieure, l'humeur vitrée - ici nue, découverte - rencontre le cristallin (mais $c f$. ci-dessous) ${ }^{26}$.

Le corps sphérique antérieur dans l'œil, plus petit et semblable à de la glace (le cristallin : fig. a, 7), est légèrement aplati ou elliptique ${ }^{27}$. Sa face

26. $P H P$ 7.5.25-26 (V, p. 623-624 K. $=458$ De Lacy). Chez Galien, la forme de l'interface entre l'humeur vitrée et le cristallin est relativement claire (voir ci-dessous, IV), mais sa nature matérielle ne l'est pas. Dans Anat. adm. 10.3 (p. 48-52 Simon = 40-42 D. = 871-874 G.), qui ne subsiste qu'en arabe, Galien suggère p. ex. qu'une gaine délicate

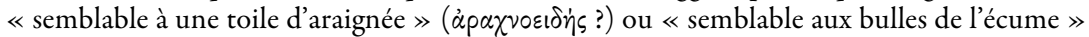
(å $\phi \omega \dot{\delta} \delta \eta \varsigma$ ?, écumeuse) « enveloppe le cristallin à l'extérieur ». Certains médecins, nous dit Galien, « comptent cette gaine comme une des tuniques ( $\chi(\tau \tilde{\omega} v \varepsilon \varsigma$ ?) de l'œil, parce qu'elle entoure et habille le cristallin » (p. 48 Simon). Galien ajoute cependant par la suite quelques remarques qui créent au moins autant de problèmes qu'elles n'en résolvent. D'abord, il affirme qu' «il est tout à fait correct de dire que la substance qui a cette structure [écumeuse] ne se répand qu'autour des régions du cristallin avec lesquelles l'humeur vitrée est en contact direct, et de décrire comme une gaine délicate ce qui se trouve au creux situé dans la tunique semblable à un grain de raisin » (c.-à-d. dans l'iris-uvée, à la pupille) (p. 48 Simon). Deuxièmement, il dit que « [la gaine] qui habille le cristallin sur sa face postérieure [c.-à-d. la capsule postérieure du cristallin est... comparable à ce que les Grecs appellent

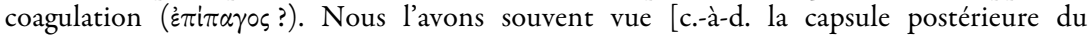
cristallin ?] plus épais(se) que ce qui enveloppe l'humeur vitrée, à un degré comparable à celui auquel ce qui enveloppe l'humeur vitrée est plus fin que la structure qui entoure le cristallin à la pupille. Et souvent aussi, nous avons trouvé qu'elle ressemblait à la paroi membraneuse distendue d'une bulle d'écume. Par conséquence, on utilise l'expression correcte en appelant cela [la capsule postérieure du cristallin] une tunique cystique [vésiculaire], et ce qui se trouve devant [la capsule antérieure du cristallin] une gaine arachnoïde. Car ce qui forme la paroi de la bulle est beaucoup plus fin qu'une toile d'araignée » (p. 49 Simon). Les ambiguités de ce passage - et il y en a bien davantage, que je passe ici sous silence - sont-elles dues à Galien ou à son traducteur arabe ? Il est impossible d'en décider. Selon UP 10.6 (III, p. 787 K. = II, p. 75 Helmr.), cependant, la « tunique propre [du cristallin] ne ressemble pas seulement à la pelure d'un oignon sec [cf. Odyssée 19. 232-233], mais elle est encore plus ténue et plus claire que les fines toiles d'araignée, et, ce qu'il y a de plus remarquable, c'est qu'elle ne s'étend pas autour de tout le cristallin, qui est complètement dépourvu de protection, privé de tunique là où il s'unit à l'humeur vitrée ». Sur l'humeur vitrée, Galien dit, non qu'elle est une tunique, mais simplement que sa section extérieure est plus compacte et condensée que ses parties intérieures, plutôt qu'elle n'est une tunique (Anat. adm. 10.3, p. 48-49 Simon).

27. Galien ne nomme cette forme lenticulaire ou lentiforme que dans des passages conservés uniquement par la traduction arabe, p. ex. Anat. adm. 10.3 (p. 48 Simon = p. 39 D.

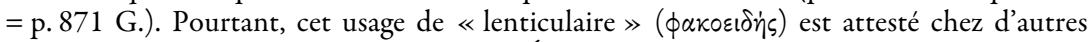
auteurs de textes médicaux grecs : Rufos d'Éphèse, De nominatione partium hominis, 153 (p. 154.12 Daremberg/Ruelle); Rufos (?), De dissectione partium hominis, 16 (p. 172.6 
postérieure s'appuie directement sur la face antérieure légèrement concave de l'humeur vitrée. Les explications téléologiques que donne Galien de cette interface seront examinées ci-dessous (IV). Anatomiquement, cependant, Galien explique l'absence de ces trois tuniques de la face antérieure de l'humeur vitrée par le fait que les extrémités antérieures de ces tuniques sont toutes insérées dans ce que Galien appelle «le plus grand cercle du cristallin $\gg^{28}$, c.-à-d. dans ses bords latéraux, là où le cristallin, légèrement elliptique, possède la plus grande circonférence. Toutes ces trois membranes, en d'autres termes, cessent de couvrir l'humeur vitrée à ses bords latéraux.

Alors que la face postérieure du cristallin n'est apparemment recouverte par aucune «tunique » - bien que les affirmations de Galien sur cette question soient loin d'être dépourvues d'ambiguïté (voir n. 27) -, sa surface antérieure est partiellement recouverte par différentes couches. La première, située directement devant le cristallin, nous dit Galien, est une tunique extrêmement fine, semblable à une toile d'araignée ou « arach-

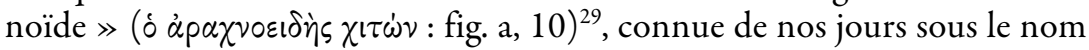
de « capsule du cristallin ». Sur son extérieur, la capsule est suivie de l'humeur aqueuse ou « fine » (ípó̀ns $\tau$ เs $\lambda \varepsilon \pi \tau \dot{\eta}$ : fig. a, 11), qui se trouve entre le cristallin et le côté postérieur de l'iris ${ }^{30}$. Galien semble avoir appelé l'iris (fig. a, 12) dans son ensemble « la membrane semblable à un grain de

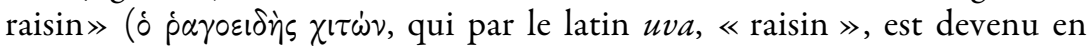
français « uvée » et en anglais « uvea », utilisées principalement de nos

Daremberg/Ruelle); Aëtios d'Amide, 7.1 (CMG VIII.2, p. 254.19 Olivieri). Sur l'instabilité de la nomenclature grecque antique pour les tuniques des yeux, voir Lloyd 1983, p. 161.

28. D'après Galien, la jonction de la sclérotique et de la cornée se trouve à la couronne ou aux bords latéraux du «plus grand cercle [circonférence] du cristallin ». Parce qu'il a observé sept tuniques ou gaines insérés dans le cristallin à cette jonction - la rétine, la choroïde, la sclérotique, la cornée, l'uvée, la tunique arachnoïde et l'aponévrose qui entoure la musculature de l'œil - il considère cette jonction comme un pivot dans la structure de l'œil et comme une preuve de l'importance capitale du cristallin en tant qu'instrument principal de la vision. Voir p. ex. PHP 7.5.26 (V, p. $624 \mathrm{~K} .=$ p. 458.22 De Lacy) ; UP 10.1, 10.2 (III, p. 760, 766 K. = II, p. 55, 59-60 Helmr.) ; Anat. adm. 10, 1-3 (p. 36-37, 39-40, 4245, 47-48 Simon = p. 30-31, 33-37, 39-40 D. = p. 860-861, 863-864, 866-868 G.), notamment p. 48 Simon : «Par le biais de sa plus grande circonférence, le cristallin entre en relation avec la couronne » (c.-à-d. avec la jonction scléro-kératique). Pour plus de références, cf. supra n. 22.

29. Anat. adm. 10.3 (p. 48-51 Simon = p. 40-41 D. = p. $871-873$ G.) ; De methodo medendi, 1.6 (X, p. 47 K.).

30. UP 10.4-6 (III, p. 781-782, 784-785 K. = II, p. 70, 71, 73-74 Helmr.); Anat. adm. 10.2 (p. 42 Simon = p. 35 D. = p. 866 G.) ; De symptomatum causis, 1.2 (VII, p. 89-90, 93$94,96 \mathrm{~K}$.$) .$ 
jours pour ne désigner que la couche postérieure pigmentée de l'iris, plutôt que la totalité de l'iris) ${ }^{31}$.

Dans l'iris se trouve le célèbre orifice connu sous le nom de pupille

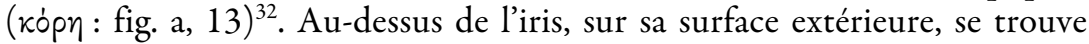
une quadruple tunique, plus dure, fine, transparente, et translucide, « la

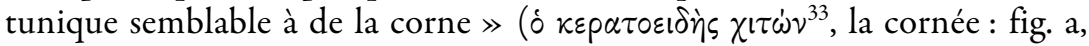
14), qui, à la différence de la « tunique semblable à un grain de raisin » bleutée (c.-à-d. de l'iris : fig. a, 12), est claire et transparente. Tout comme les extrémités des trois tuniques de l'humeur vitrée, les extrémités des trois enveloppes du cristallin - la cornée, l'iris et la capsule du cristallin («l'arachnoïde ») - sont elles aussi toutes insérées dans «le plus grand cercle $\gg d u$ cristallin ${ }^{34}$. Dans le cristallin sont donc ancrées toutes les

31. Anat. adm. 10.2, 10.3 (p. 42-43, 45, 53 Simon = p. 35-36, 37, 43 D. $=$ p. $866-867$, 868,875 G.). Galien explique que le choix du terme « semblable à un grain de raisin » pour faire référence à cette tunique vient du fait qu'elle a un aspect lisse à l'extérieur, mais que sa surface profonde est rugueuse. Certains l'appellent, dit-il, « la couche perforée », à cause de son orifice central (c.-à-d. la pupille) : voir n. 33. L'uvée est décrite comme libre de la cornée et du cristallin, ne leur étant liée qu'à la jonction scléro-kératique. Voir aussi UP 10.4, 10.56, 10.15 (III, p. 779, 781-783, 785-787, 839 K. = II, p. 69, 71-75, 111 Helrm.) ; PHP 5.3.6, 7.4.12, 7.4.15, 7.6.28 (V, p. 446, 614, 615, 635 K. = p. 306, 450, 468, De Lacy); De morborum causis, 1.2 (VII, p. 87-98 K.); De methodo medendi, 1.6 (X, p. 47 K.); De compositione medicamentorum secundum locos, 4. 1-2, 4.4 (XII, p. 705, 709, $716 \mathrm{~K}$.).

32. Cet orifice est circulaire, observe Galien, chez de nombreux animaux, mais en forme de fente pour le bétail. À travers l'ouverture (la pupille), on peut voir l'humeur semblable à de la glace (le cristallin) et sa propre image dans le cristallin, « comme si l'on regardait dans un miroir » : Anat. adm. 10.2 (p. 44 Simon = p. 37 D. $=868$ G.). Voir aussi Anat. adm. $10.1,10.3$ (p. 36, 48-49, 51 Simon = p. 30, 40, 41 D. = p. 860, 871-872, 873 G.) ; UP 8.6, 10. 4-6, 10.8, 10.13, 10.15 (III, p. 644, 779-788, 795-796, 830-831, 839-840 K. = I, p. 467 ; II, p. 69-76, 81, 105, 111-112 Helmr.) ; PHP 7.4.11-12, 7.4.15, 7.4.18-19, 7.5.2, 7.7.8 (V, p. 614-616, 618, 639 K. =450, 452, 470 De Lacy). Cf. Ars medica, 9 (I, p. 330 K.); De symptomatum causis, 1.2 (VIII, p. 87-101 K.) ; De locis affectis, 1.2, 4.2 (VIII, p. 22-23, 222225, 228 K.) ; De morborum differentiis, 8, 13 (VI, p. 862, 877 K.); In Hippocratis Prognosticum comm. 1.23 (XVIIIB, p. 71-74 = CMG V 9, 2, p. 236-237 Heeg).

33. Anat. adm. 10.1-2 (p. 37, 40-45 Simon = p. 31, 34-36 D. = p. 861-862 G.), la cornée est blanche; ses quatre laminae ou «feuilles » peuvent être ôtées, d'après Galien, à l'aide d'un scalpel en forme de feuille de myrte, et chacune des quatre couches a des caractéristiques distinctives qu'elle conserve en chaque endroit de la cornée. Les quatre feuilles de la cornée adhèrent toutes fermement à la jonction scléro-kératique. Sur la cornée, voir aussi UP 8.6, 10.1, 10.3-7 (III, p. 646-644, 760-761, 771-774, 778-785, 787-789, 791-793 K. = I, p. 466-467 ; II, p. 55, 64-66, 68-76, 78-79 Helmr.); PHP 5.3.6 (V, p. $446 \mathrm{~K} .=306 \mathrm{De}$ Lacy); De morborum differentiis, 13 (VI, p. 876-878 K.) ; De morborum causis, 10 (VII, p. 36 K.) ; De symptomatum causis, 1.2 (VII, p. 87-88, 94, 98-101 K.) ; Ad Glauconem de methodo medendi, 2.1 (XI, p. 77 K.) ; De methodo medendi, 1.6 (X, p. 47 K.) ; De compositione medicamentorum secundum locos, 4. 1-2, 4.4 (XII, p. 705, 709, 716 K.) ; In Hippocratis Prognosticum comm. 1.23 (XVIIIB, p. 71-72 K. = CMG V 9, 2, p. $236 \mathrm{Heeg}$ ).

34. Voir supra n. 28. 
enveloppes à la fois du corps sphérique vitré et du corps sphérique cristallin (aussi bien que la gaine couvrant la musculature de l'œil) - fait qui, à un niveau anatomique, signale la primauté du cristallin, selon Galien.

Pour Galien, l'anatomie n'est évidemment pas seulement un moyen d'observer par la dissection ou vivisection afin d'établir une description détaillée et définitive des parties du corps ; l'anatomie est aussi un point de départ épistémologique indispensable pour une science du corps, c.-à-d. pour l'explication téléologique de chaque partie du corps humain. Plus particulièrement, ses observations anatomiques de l'œil, du nerf optique, des nerfs et muscles oculomoteurs, des veines et artères de l'œil, et des ventricules d'encéphale, souvent accompagnées d'expériences de vivisection, ont fourni un soutien capital à sa théorie de la vision et à ses affirmations répétées qu'il a posé des fondements observationnels et expérimentaux qui garantissent la supériorité de sa théorie sur celles de ses précurseurs. Deux exemples d'expériences célèbres de vivisection effectuées par Galien dans ce cadre méritent d'être cités ici. Ayant observé que deux paires de nerfs s'avancent du cerveau vers l'œil, Galien remarque :

Si l'on sectionne le plus large des deux nerfs qui vont vers l'œil [c.-à-d. le nerf optique], la perception visuelle de l'animal [vivant] sera détériorée ; mais si l'on sectionne le plus petit des deux [c.-à-d. le nerf oculomoteur], on verra que l'œil reste immédiatement immobile. Le défaut de mouvement oculaire [causé en sectionnant le nerf oculomoteur] est un signe que l'on peut aisément détecter... Mais que l'animal ne puisse plus voir dans cet état de blessure qu'on lui a infligée [en sectionnant le nerf optique], est une chose que l'on ne peut déterminer qu'en l'inférant du fait suivant : si l'on approche un objet de son œil, en faisant semblant de vouloir le frapper à l'œil avec l'objet, il ne cligne pas de l'œil ${ }^{35}$.

Cette expérience, comme beaucoup d'autres, permet à Galien de passer d'une anatomie descriptive à une anatomie fonctionnelle, qui à son tour ouvre la voie à la physiologie de la vision (ci-dessous, III).

De même, une autre expérience faite sur un animal vivant permet à Galien d'établir des rapports fonctionnels entre l'œil et les ventricules antérieurs d'encéphale ${ }^{36}$. Si l'on ouvre le crâne, dit-il, qu'on dénude le cerveau de la dure-mère et que l'on comprime ou incise le quatrième ventricule (ventricule postérieur), on cause ainsi, normalement, trop de dommages pour permettre à l'animal de revenir jamais à son état normal. Néanmoins, si l'incision est rapide, petite, et faite par une main experte, alors une compression très brève et adroite du quatrième ventricule peut permettre à

35. Anat. adm. 10.1 (p. 35 Simon = p. 29 D. $=$ p. 859 G.).

36. Anat. adm. 9.12 (p. 21-24 Simon = p. 17-20 D. = p. $845-847$ G.). 
l'animal de se remettre. C'est dans ce contexte que Galien aborde les rapports entre la vision et les ventricules du cerveau :

On voit que même lorsque l'on a exposé aux regards le ventricule postérieur [le quatrième] du cerveau, l'animal cligne encore des yeux, surtout si l'on approche quelque objet de ses yeux. Par contraste, si l'on prend un animal dans cet état et que l'on comprime une partie des deux ventricules antérieurs - quelque partie que ce puisse être - à la racine des deux nerfs optiques, dans ce cas, l'animal cesse de cligner des yeux, même lorsque l'on approche un objet de la pupille de ses yeux. Et tout l'aspect de l'œil sur le côté du ventricule du cerveau sur lequel on appuie devient semblable à celui des yeux des aveugles ${ }^{37}$.

Cette expérience prouve, alors, que les fonctions des deux ventricules antérieurs (latéraux) du cerveau - mais pas celles du ventricule postérieur dans la moelle allongée (myélencéphale) - comportent de quelque façon une responsabilité dans le mécanisme de la vision. Tout comme l'expérience de vivisection sur les nerfs optique et oculomoteur décrite ci-dessus, cette expérience a ainsi conduit à une importante découverte qui a ouvert la voie à la description physiologique de la vision de Galien.

Une autre stratégie heuristique et justificatrice qui fait son apparition dans les discussions anatomiques de la vision est la morphologie comparative. Dans les six catégories d'animaux, certains organes diffèrent morphologiquement d'une catégorie à l'autre, observe Galien, tandis que d'autres montrent des caractéristiques constantes à travers ces catégories ${ }^{38}$. L'œil appartient à ce dernier groupe : à l'exception de sa taille et de la forme de certaines de ses parties, comme la pupille, l'œil fait preuve d'invariabilité. D'une espèce vertébrée à l'autre, la structure de base de l'œil, le nombre et l'agencement de ses parties, ses activités et ses fonctions montrent une invariabilité, comme le confirme, dit Galien, l'observation anatomique ${ }^{39}$.

37. Anat. adm. 9.12 (p. 23-24 Simon = p. 19 D. = p. 845-847 G.). Parmi d'autres utilisations d'animaux pour l'étude de l'œil, Galien décrit comment observer et disséquer les petits vaisseaux sanguins liés à la sclérotique et qui en partent pour la tunique choroïde de l'œil, sans séparer aucune de ces deux tuniques de l'humeur vitrée. Il faut tenter cette expérience avec un animal tué il y a peu, qui n'a pas été vidé de tout son sang: par exemple, «s'il arrivait qu'un cheval, non affecté par la maladie, s'effondre à la suite d'une longue course (et cela arrive fréquemment), essayez de dégager ces vaisseaux sanguins sur un tel spécimen... [ou] suffoquez un animal jusqu'à ce qu'il meure, soit au moyen d'un nœud coulant, soit en le maintenant immergé sous l'eau, et vous obtiendrez des informations sûres sur ces petites veines », à la fois dans l'œil et ailleurs (Anat. adm. 10.2, p. 45-46 Simon $=$ p. 38 D. $=$ p. 869 G. $)$.

38. Anat. adm. 10.1 (p. 34 Simon = p. 29 D. = p. 859 G.). Voir Garofalo 1991 b.

39. Anat. adm. 10.1 (p. 34 Simon = p. 29 D. = p. 859 G. $):$ « L'œil fait partie de ces organes dont la forme est la même » à travers toutes les catégories d'animaux et, « comme le 
L'invariabilité de la structure, au-delà des différences d'espèces, et l'unicité d'un grand nombre de caractéristiques de l'œil, sont ainsi des fils conducteurs de l'anatomie descriptive et fonctionnelle de l'œil, remarquablement détaillée, chez Galien. En plus de l'œil lui-même, Galien décrit d'autres parties qui sont plus ou moins pertinentes pour sa théorie de la vision. Par exemple, il fait une description détaillée des nerfs oculomoteurs qui descendent par les foramina orbitorunda crâniens du cerveau jusqu'aux muscles des yeux, aussi bien que de presque tous les muscles qui font bouger les paupières, les cils et la peau du visage ${ }^{40}$. De même, il développe amplement la structure anatomique des paupières, des cils, des coins des yeux, des glandes lacrymales, et des artères et veines qui arrivent dans l'espace orbitaire de chaque oil $^{41}$. Le bref aperçu anatomique ci-dessus donne cependant, on l'espère, un contexte anatomique suffisant pour aborder l'analyse de la physiologie de la vision chez Galien, c.-à-d. de sa théorie de l'utilité (chreia), du pouvoir (dynamis, puissance, faculté), de l'activité (energeia) et de la fonction (ergon) de chacune de ces parties de l'instrument (organon) de la vue.

\section{La physiologie de la vision : pneuma, transmission de puissance et altération}

En se référant à Aristote, Galien affirme que l'essence d'un organisme vivant est à trouver non dans ses parties, mais dans ses activités ${ }^{42}$. La connaissance anatomique des parties de l'organe visuel est donc un pas nécessaire mais non suffisant vers un projet plus significatif : la connaissance de ses activités essentielles.

Peu de concepts sont aussi fondamentaux pour l'explication galénique de l'activité essentielle de l'œil - la vue - que le pneuma. Le pneuma, et notamment le «pneuma psychique » ou «pneuma appartenant à l'âme » $(\pi v \varepsilon \tilde{v} \mu \alpha \psi \nu \chi\llcorner\kappa o ́ v)$, est une pierre angulaire non seulement de la théorie de la

montre l'étude du pouvoir du nerf optique, à cet égard les différents animaux sont tous pareils et concordent ».

40. Par ex., Anat. adm. 9.8, 9.9, 10.1, 10.4, 14.2, 14.4 (p. 7, 9-11, 34-40, 54-64, 235-238, 246-247 Simon = p. 6, 8-9, 28-33, 43-51, 186-188, 195-196 D. = p. 831-832, 834-835, 859864, 876-884, 1044-1046, 1053-1055 G.) ; UP 9.8, 9.13, 10.6-10, 11.14-15, 17.1 (III, p. 712-713, 716-719, 732-733, 789-808, 901-913 ; IV, p. 356-358 K. = II, p. 22, 25-26, 3637, 77-90, 155-164, 445 Helmr.) ; PHP 6.3.32-35 (V, p. 529-530 K. = 380-382 De Lacy); De nervorum dissectione, 3 (II, p. $833 \mathrm{~K} .=$ p. 28 Garofalo et Debru).

41. Voir n. 41 ; UP 10.11 (III, p. 809-812 K. = II, p. 90-92 Helmr.).

42. Par exemple, PHP 1.8.9-15 (V, p. 202-203 K. = 92-94 De Lacy). Voir aussi Galien, De partium homoeomerum differentiis, 2 (CMG Suppl. Orient. III, p. 53). Cf. Aristote, De partibus animalium, 1, 1640b23-1641a6; De anima, II, 1, 412b10-22 ; 4, 416a4-5 ; Pol. I, 2 , $1253 \mathrm{a} 23$. 
vision de Galien, mais aussi de sa théorie de toute sensation, perception et mouvement volontaire. Cependant, la notion de pneuma psychique semble, paradoxalement, avoir été reprise par Galien de l'une des principales cibles de ses polémiques, à savoir Érasistrate (à qui Galien, en dépit de ses nombreux écrits anti-érasistratéens, a emprunté nombre de ses principales doctrines physiologiques, tout en étant, sous certains aspects, débiteur d'Hérophile de manière significative en ce qui concerne l'anatomie $\left.{ }^{43}\right)$. En désaccord sur les causes et les mécanismes par lesquels ce phénomène arrive, Érasistrate et Galien s'accordaient à reconnaître, en premier lieu, que tout le pneuma ou «souffle » dans le corps provient en fin de compte de l'air extérieur par le biais de la respiration; deuxièmement, qu'une partie de l'air inspiré subit des modifications d'abord dans les poumons, puis dans le ventricule gauche du cœur; et troisièmement, que les artères amènent alors

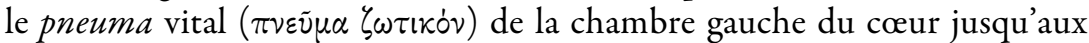
ventricules $($ col $\lambda i \alpha \iota)$ du cerveau ${ }^{44}$. C'est là, dans le cerveau, que le pneuma vital devient à son tour élaboré ( $\kappa \tau \tau \varepsilon p \gamma \dot{\alpha} \zeta \varepsilon \tau \alpha \iota)$ ou raffiné en pneuma psychique, en partie grâce aux circonvolutions ou « filets » vasculaires extensifs; deux de ces réseaux - le plexus réticule et le plexus choroïde assurent au cerveau sa vascularisation exceptionnelle, d'après Galien ${ }^{45}$.

43. Sur Galien et Érasistrate, voir Debru 1996, p. 53-60, 133-137; Garofalo 1988, notamment p. 8-15 ; Hankinson 1998, p. 30-35, 105-109, 117-137, 224-227 ; Roselli 1999; Vegetti 1999 ; Cambiano 2000. Sur Hérophile, voir supra n. 21 et p. ex. Galien, Anat. adm. 3.2, 6.8, 6.9, 9.1, 9.3, 9.5, 9.9, 10.4, 10.7, 11.1, 12.2, 12.8, 13.1, 14.5 (II, p. 349, 570-572, 712, 719, 731, K. ; p. 12, 59, 70, 84, 141, 164, 173, 252-253 Simon = p. 10, 48, 56, 88, 112, 131, 138, 201 D. = p. 836, 880, 889, 903-904, 956, 976, 987, 1059-1060 G.); De venarum arteriarumque dissectione, 1.6 (II, p. 780-781 K. = p. 77 Garofalo et Debru) ; UP 4.19, 6.10, 8.11, 9.6, 10.12, 14.3, 14.11 (III, p. 335, 445, 665-667, 708, 813 ; IV, p. 149, 190, $193 \mathrm{~K}$. = I, p. 246-247, 325, 482-484; II, p. 19, 93, 290, 321, 323 Helmr.) ; De semine, 1.16.5, 2.1.15-23, 2.6.13 (IV, p. 582, 596-598, 646 K. = CMG V 3.1, p. 134, 146-148, 200 De Lacy); In Hippocratis Epidemiarum, 2.4.1-2 Comm. 4 (CMG V 10.1, p. 312, 318, 322, 330 Pfaff) ; De locis affectis, 3.14, 6.3 (VIII, p. 212, 396 K.) ; De symptomatum causis, 1.2 (VII, p. 88-89 K.) ; De uteri dissectione, 3, 5, 7 (CMG V 2.1, p. 38, 43-44, 46 Nickel) ; De pulsuum usu, 2 (V, p. 155 K. = p. 200 Furley/Wilkie). Cf. von Staden 1991, sur d'autres traits des rapports de la pensée de Galien avec celle d'Hérophile.

44. PHP 1.6.11, 3.8.29-32, 7.3.19-30, 8.8.7 (V, p. 187, 355-356, 606-609, 708-709 K. $=80,230,442-446,528$ De Lacy); UP 7.8, 7.9, 8.10, 8.11, 9.4 (III, p. 541-542, 544-545, 663, 665, 698-703 K. = I, p. 393-394, 396, 481, 482; II, p. 11-15 Helmr.). Cf. De usu respirationis, 5 (IV, p. 501-511 K. = p. 120-132 Furley/Wilkie); De usu pulsuum, 2 (V, p. 153-156 K. = p. 198-202 Furley/Wilkie). Voir Debru 1996, notamment p. 145-163.

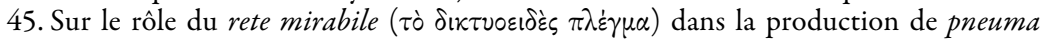
psychique, voir p. ex. PHP 3.8.31, 7.3.23-29 (V, p. 356, 606-609 K. =230, 444-446 De Lacy) ; UP 7.8, 9.4, 16.10 (III, p. 541-542, 696-702 ; IV, p. 323 K. = I, p. 393-394 ; II, p. 1014, 420 Helmr.); von Staden 1989, p. 158-159, 179, 223 (fr. 121); Debru 1996, p. 156 (plexus réticulé); Harris 1973, p. 354-362 ; Rocca 2003, p. 202-219. Sur le pneuma vital et 
Tout comme les nerfs, le pneuma psychique se divise en deux catégo-

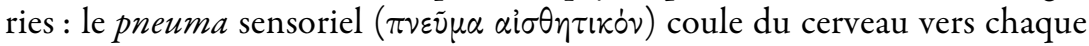
organe des sens par les nerfs sensoriels, qui sont relativement mous, alors

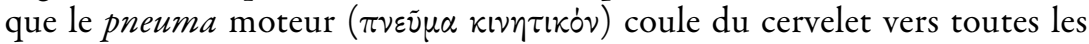
parties du corps par les nerfs moteurs, qui sont plus durs, permettant aux muscles des mouvements volontaires ${ }^{46}$. Il va peut-être sans dire que sa connaissance anatomique détaillée du cerveau et du système nerveux amène Galien à se montrer très critique vis-à-vis des théories de perception cardiocentristes, et qu'il critique longuement Aristote et les stoïciens pour avoir

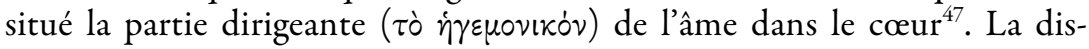
section, et en particulier les expériences de vivisection sur les animaux (cidessus, II), affirme Galien, prouvent au-delà du doute que le cerveau est le centre d'où coulent tous les pouvoirs sensoriels et moteurs de l'âme.

Dans la théorie de la vision de Galien, comme dans n'importe laquelle de ses doctrines physiologiques, le rapport entre l'observation anatomique et l'explication physiologique est étroit et d'une importance capitale. Comme on l'a signalé ci-dessus (II), selon lui, partout où l'on observe l'unicité anatomique, la fonction et la finalité physiologiques doivent être considérées comme uniques. Dans le cas de la vision, le conduit ( $\pi$ ópos: fig. a, 4) perceptible et exceptionnellement large à l'intérieur du nerf optique sert à permettre à une quantité inhabituellement grande de pneuma psychique sensoriel de couler des deux ventricules antérieurs du cerveau jusque dans l'œil $^{48}$. De plus, le degré unique de mollesse des nerfs optiques (ils sont $\mu \alpha \lambda \alpha \kappa \dot{\omega} \tau \alpha \tau \alpha)$ sert à les rendre exceptionnellement susceptibles d'être affectés $^{49}$. De manière générale, la dureté des nerfs moteurs les rend capables

le pneuma psychique, voir Harris 1973, p. 336-363 ; Debru 1996, p. 145-160 ; Manzoni 2001, p. 29-61 ; Rocca 2003, p. 59-66.

46. Voir supra n. 10 et De symptomatum causis, 1.2 (VII, p. 88-89 K.); De locis affectis (VIII, p. 174-175 K.). Cf. PHP 3.6.3, 7.5.13-16, 7.5.29, 8.1.1 (V, p. 333, 621-622, $624 \mathrm{~K}$. $=210,456,458$ De Lacy).

47. P. ex. PHP 2.2-8, 3.1-8 (V, p. 213-359 K. = 102-233 De Lacy).

48. Voir supra n. 11, surtout UP 8.6 (III, p. 641-642 K. = I, p. 465 Helmr.) : « Cette

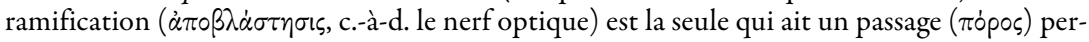
ceptible, parce que c'est la seule [parmi tous les nerfs] qui contienne une très grande quantité de pneuma psychique. » Aussi PHP 7.5.19-20 (V, p. $622 \mathrm{~K} .=456-458$ De Lacy) : « C'est par conséquent pour une bonne raison que de tous les nerfs - pas seulement ceux qui se sont développés à partir du cerveau, mais aussi ceux qui l'ont fait à partir de la moelle épinière -, c'est celui de la vision qui a été créé le plus large. Car c'était pour posséder des traits exceptionnels, allant au-delà de ceux du reste des nerfs : ... une grande quantité de substance pneumatique devait [passer] à travers lui en partant du cerveau [et] arriver jusqu'aux espaces des yeux. »Voir aussi $P H P$ 7.5.30 (V, p. $625 \mathrm{~K} .=458$ De Lacy).

49. $P H P$ 7.5.15-17 (V, p. 621-622 K. = 456 De Lacy); UP 16.3 (IV, p. $278 \mathrm{~K} .=\mathrm{II}$, p. 385 Helmr.). Cf. UP 8.5 (III, p. 633 K. = I, p. 459 Helmr.): « Chacun de ces 
d'agir ( $\pi \circ \varepsilon \tilde{\varepsilon} \nu, \delta \rho \tilde{\alpha} \nu)$, tandis que la consistance molle des nerfs sensoriels les rend capables d'être affectés $(\pi \dot{\alpha} \sigma \chi \varepsilon เ \nu)$. L'exceptionnelle mollesse du nerf optique fait donc de lui le plus capable d'altération et le plus sensible à l'altération $\left(\dot{\alpha} \lambda \lambda \lambda_{0} \omega \omega \sigma \zeta\right)^{50}$, ce qui lui permet non seulement de subir mais aussi de transmettre l'altération, et ainsi de transmettre les détails concer-

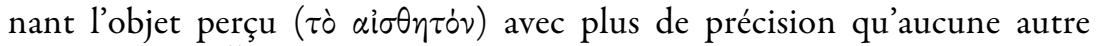
partie du corps ${ }^{51}$.

Ces capacités ou pouvoirs ( $\delta v v \alpha \dot{\alpha} \mu 1 \varsigma)$ uniques du nerf optique ne sont pas limités à la partie qui se trouve entre le cerveau et l'œil (fig. a, 3) mais s'étendent aussi à la rétine (fig. a, 5) tout du long jusqu'à son insertion dans le cristallin (fig. a, 7). Après tout, la rétine tout entière, en tant qu'extension aplatie et étendue du nerf optique (fig. a, 3), est elle-même à la fois nerf et extension de la substance même du cerveau $(c f \text {. II })^{52}$. C'est précisément pour protéger ces capacités inhabituelles, dit Galien, que le nerf optique et la rétine sont tous deux recouverts par les deux tuniques plus dures qui sont des continuations respectivement de la pie-mère et de la dure-mère ${ }^{53}$.

Au moins deux sortes de matière différentes arrivant du cerveau à l'œil apparaissent ainsi jouer un rôle dans la vision: (1) le pneuma psychique sensoriel, qui coule des ventricules antérieurs (latéraux) du cerveau jusque dans l'œil par le conduit creux situé dans le nerf optique ; et (2) la substance matérielle du cerveau, étendue finalement jusqu'à l'œil sous la forme de la rétine. Parmi plusieurs problèmes difficiles que présente la physiologie de la vision de Galien se trouvent, d'abord, l'identification précise de la fonction attribuée à chacune de ces deux connexions matérielles entre l'œil et le cerveau et, ensuite, la précision des rapports entre leurs fonctions respectives.

À propos de la finalité de la rétine, Galien dit, par exemple: «La première et plus grande utilité, en vue de laquelle elle [scil. la rétine] est envoyée du dessus [scil. du cerveau], c’est de percevoir les altérations éprouvées par l'humeur cristalline et en outre d'apporter et transmettre à l'humeur vitrée son aliment $\mathrm{t}^{54}$. $\gg$ Cette dernière finalité - la transmission de

instruments des sens a besoin d'un nerf mou : un nerf, parce que c'est l'instrument (öp $\gamma \alpha \nu \circ v)$ de la perception ( $\alpha$ lै $\theta \eta \sigma \iota \zeta)$, et mou, parce que l'instrument du sens doit être en quelque façon disposé ( $\delta \iota \tau \tau \varepsilon \theta \tilde{\eta} \nu \alpha \iota)$ et affecté ( $\pi \alpha \theta \varepsilon i v)$ à quelque égard par ce qui est tombé du dehors contre lui, afin que la perception puisse avoir lieu. Et ce qui est mou ( $\mu \alpha \lambda \alpha \kappa o \dot{v})$ est plus propre à être affecté, ce qui est dur à agir [sur quelque chose]. » Voir aussi De nervorum dissectione, 2 (II, p. 832-833 K.) et supra n. 9, 10 et 13.

50. UP 16.3 (IV, p. 272-276 K. = II, p. 382-385 Helmr.).

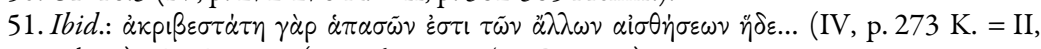
p. 383 Helmr.); PHP 7.5.29 (V, p. $625 \mathrm{~K} .=458$ De Lacy).

52. PHP 7.5.17-18, 7.5.29-30 (V, p. 622, 625 K. = 456, 458 De Lacy). Voir supra n. 17.

53. Voir supra n. 7.

54. UP 10.2 (III, p. $762 \mathrm{~K}$. = II, p. 56-57 Helmr.). 
nourriture au corps sphérique postérieur - est effectivement accomplie, dit-il, par de nombreuses petites branches d'artères et de veines insérées dans la rétine à partir de la membrane choroïde du cerveau ${ }^{55}$, « car avec tous les nerfs issus du cerveau se détache une partie de la membrane choroïde [ du cerveau], amenant une artère et une veine ; mais aucun des autres nerfs n'est accompagné de vaisseaux aussi considérables » que l'est le nerf optique $^{56}$. Pour ce qui est de l'autre finalité de la rétine - percevoir les altérations dans l'humeur cristalline - on essaiera ci-dessous d'expliquer la

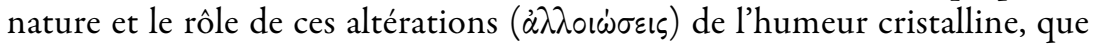
Galien nomme souvent « l'instrument principal de la vision ».

Mais d'abord il sera utile d'examiner brièvement quelques questions soulevées par l'affirmation galénique citée à l'instant : si, comme le soutient Galien, la rétine elle-même est responsable de la perception ( $\alpha i \sigma \theta \dot{\alpha} \nu \varepsilon \sigma \theta \alpha \iota)$, et si la rétine, en tant qu'extension de la substance molle du nerf optique et conséquemment de la substance matérielle du cerveau lui-même, est une continuation de la matière du cerveau, alors quelle est la fonction du pneuma psychique? En particulier, le pneuma optique communique-t-il quelque chose en retour de l'œil aux ventricules antérieurs du cerveau, et si oui, comment ? Un rapide examen des remarques dispersées de Galien sur le pneuma optique est un préliminaire nécessaire à toute réponse à ces questions.

Que se passe-t-il lorsque la grande quantité de pneuma optique qui coule du cerveau en passant par le nerf optique arrive à l'œil ? D'après Galien, ce pneuma se mêle à une série de trois corps oculaires humides contigus, passant successivement de l'arrière à l'avant de l'œil. D'abord, il se

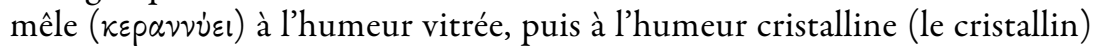
immédiatement adjacente, puis à l'humeur aqueuse (fig. a, 11) qui se trouve entre le cristallin et l'iris ${ }^{57}$. Le mélange du pneuma avec chacun de ces trois corps s'effectue en profondeur, et le but du mélange est d'assurer en particulier la capacité d'aisthesis à la substance entière de chacun des deux corps

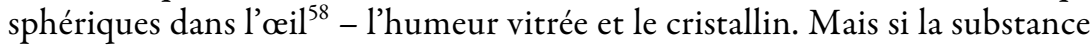

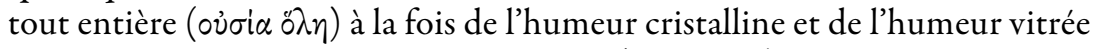
est capable de sensation et de perception ( $\alpha i \sigma \theta \eta \tau \leftarrow \kappa \eta)$, et si ce pouvoir sensible est une continuation du pneuma psychique de variété sensorielle dans les ventricules cérébraux, et se trouve donc par là en contact ininterrompu

55. Ici « la membrane choroïde » signifie le repli membraneux qui forme la pie-mère dans les ventricules latéraux (ou « antérieurs ») du cerveau, tandis qu'ailleurs Galien utilise « la membrane choroïde » pour désigner la tunique uvée de l'œil. Voir supra n. 26.

56. UP 10.2 (III, p. 762-3 K. = II, p. 57 Helmr.).

57. Voir supra n. 30.

58. PHP 7.5.26 (V, p. $624 \mathrm{~K} .=458$ De Lacy). 
avec lui, pourquoi la vision requiert-elle aussi la présence de la rétine ? Après tout, le pneuma sensoriel n'est jamais absent de l'œil, puisqu'il est produit en continu dans le cerveau à partir du pneuma vital et arrive donc sans interruption dans l'œil par le nerf optique (à part, bien sûr, dans quelques conditions pathologiques dont Galien discute dans le De symptomatum causis et le De locis affectis $\left.{ }^{59}\right)$. Les humeurs cristalline et vitrée sont en conséquence continuellement sensibles ( $\alpha i \sigma \theta \eta \tau \iota \kappa \dot{\alpha})$. Pourtant, Galien affirme aussi que «la première utilité » de la rétine est « de percevoir ( $\alpha i \sigma$ $\theta \dot{\alpha} \nu \varepsilon \sigma \theta \alpha \iota)$ les altérations $(\dot{\alpha} \lambda \lambda$ oเ $\dot{\omega} \sigma \varepsilon ı)$ ) éprouvées par le cristallin ${ }^{60}$. Comme on va le montrer ci-dessous, il se réfère ici précisément aux altérations dans le cristallin «pneumatisé » qui sont provoquées, en fin de compte, par les objets de la perception. Aucun des deux - ni la rétine en tant qu'extension de la substance du nerf optique, ni le pneuma sensoriel en tant que contenu du canal creux du nerf - n'est donc nécessaire comme suppléant compensatoire éventuel de l'autre : dans des conditions non déviantes, tous les deux sont présents en permanence dans l'œil, capables d'aisthesis en permanence. Galien ne semble pas donner de réponse claire et sans équivoque à la question de la relation entre leurs fonctions respectives, mais quelques détails supplémentaires concernant la nature et les activités du pneuma sensoriel peuvent nous aider à avancer vers une solution possible.

Si le pneuma psychique sensoriel, en sortant du nerf optique, entre de façon continue dans l'œil d'une personne éveillée, alors ce pneuma doit ou être consommé de quelque façon dans l'œil, ou ressortir de l'œil, pour que l'œil ne devienne pas excessivement distendu ni n'explose à travers ses fines enveloppes, comme un ballon devenant dangereusement trop gonflé lorsque l'on y pompe de l'air sans relâche. L'idée de Galien paraît en effet être, en premier lieu, que le pneuma optique s'échappe régulièrement des yeux à travers l'orifice de l'iris (《la tunique semblable à un grain de raisin »), c.-à-

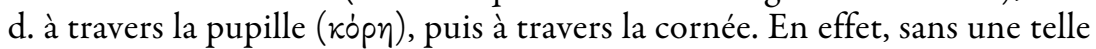
fuite du pneuma sensoriel à l'extérieur de l'œil, la vision ne pourrait avoir lieu $^{61}$. Le départ de l'un des instruments matériels, sensoriels, de l'âme hors du corps vivant pour l'espace extérieur, un trajet spatial psychique et pourtant corporel, est donc dépeint comme essentiel au bon fonctionnement du plus précis, du plus efficace, du plus privilégié épistémologiquement des cinq sens.

59. P. ex. De symptomatum causis, 1.2, 1.6 (VII, p. 86-101, 118-121 K.) ; De locis affectis, $1.2,1.7,3.1,3.3,3.9,4.2$ (VIII, p. 20-22, 66-68, 137, 139, 178, 217-229 K.). Cf. UP 10.5-6 (III, p. 784-787 K. = II, p. 72-75 Helmr.). Sur le traité galénique perdu sur les affections de l'œil, voir Savage-Smith 2002.

60. UP 10.2 (III, p. $762 \mathrm{~K} .=$ II, p. 56-57 Helmr.).

61. PHP 7.4.24, 7.5.2-7 (V, p. 617-619 K. = 452, 454 De Lacy). 
C'est principalement dans ses analyses de l'espace visuel extérieur que Galien nous donne sa version des aventures du pneuma optique hors du corps. Cet aspect de sa théorie sera examiné ci-dessous (IV) dans une analyse des fils mathématiques au sein de sa théorie ; ici, il suffit de faire remarquer qu'à son avis le pneuma sensoriel visuel s'échappant hors de la pupille produit un effet de sensibilisation instantané sur l'air extérieur qu'il rencontre dans sa sortie - effet semblable à celui produit sur l'air par la lumière du soleil :

En touchant les limites supérieures de l'air, la lumière du soleil transmet

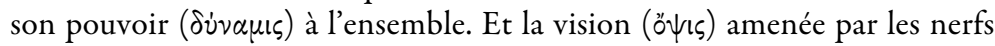
optiques contient la substance qui est pneumatique, et quand elle tombe sur l'air ambiant, elle a pour effet, par son prime impact, une altération qu'il transmet jusqu'à ce point le plus éloigné - le corps environnant étant évidemment une continuation de lui-même [c.-à-d. un continuum], de sorte qu'en un instant, il envoie l'altération à l'ensemble même ${ }^{62}$.

À première vue, il peut sembler que le cône visuel (fig. b), qui s'étend de l'œil jusqu'à l'objet de la vue, avec son sommet (A) à l'œil et sa base (BC) à l'objet visuel, consiste entièrement en pneuma sensoriel ${ }^{63}$.

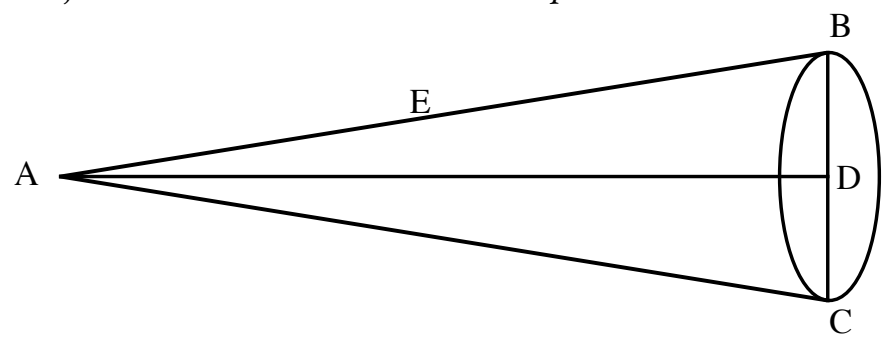

Fig. b

Galien s'exprime toutefois avec circonspection, n'identifiant pas l'air altéré par le pneuma optique explicitement comme pneuma lui-même. Plus précisément, il dit que le pneuma émis de l'œil au sommet du cône se heurte immédiatement à l'air extérieur, affectant qualitativement cet air extérieur, et pourtant dans une certaine limite :

Ainsi il est probable aussi que le pneuma qui arrive dans les yeux, à son premier impact à la fois s'unit à [l'air] environnant et l'altère qualitative-

62. PHP 7.5.7 (V, p. 619 K. = 454 De Lacy).

63. Sur le cône optique ( $\kappa \tilde{\omega} v \circ$ ), voir Gal., UP 10.12 (III, p. 815-816 K. = II, p. 95 Helmr.) ; Chrysippe, $S V F$ II, fr. 863, 864, 866, 867 ; Lejeune 1948, p. 33-34 ; Mugler 1964, p. 232-234; Hahm 1978, p. 62-67, 85, 87 ; Lindberg 1978, p. 140, 146, 153-154; Sabra 1978, p. 163-165. 
ment en lui donnant sa propre nature particulière, mais il ne s'étend pas luimême jusqu'à la distance la plus éloignée $e^{64}$.

Quand on regarde quelque chose, «l'air environnant à ce moment-là devient pour nous la sorte d'instrument (o’p $\gamma \alpha \nu \circ v$ ) qu'est le nerf dans le corps à tout moment », c'est-à-dire qu'《il transmet une puissance » qui rend la perception possible ${ }^{65}$. Peu après, Galien utilise une analogie similaire: il est probable que l'œil est construit comme il l'est parce qu'il a besoin d'utiliser l'air extérieur comme un instrument. Ainsi l'air environnant (ò $\pi \dot{\varepsilon} \rho \xi \xi \dot{\alpha} \eta \dot{\rho})$

devient pour l'œil un instrument (öpyavov) pour son propre discernement de ses objets perceptibles ( $\alpha i \sigma \theta \eta \tau \dot{\alpha})$, comme le nerf l'est pour le cerveau, en sorte que l'œil a la même sorte de rapport avec l'air [extérieur environnant] que le cerveau avec le nerf ${ }^{66}$.

La vue, nous dit Galien, est le seul sens qui utilise l'air extérieur de cette manière comme un intermédiaire au moyen duquel il perçoit l'objet sensible qui l'altère ${ }^{67}$. Tout comme son contemporain Alexandre d'Aphrodise, Galien est cependant prompt à rejeter la doctrine de Chrysippe et d'autres stoïciens selon laquelle l'étendue d'air en forme de cône entre l'œil et l'objet visuel n'est qu'un bâton ( $\beta \alpha \kappa \tau \eta p i \alpha)$ avec lequel l'œil «sent » ou examine à l'aveuglette l'objet visuel ${ }^{68}$. Bien plutôt, si la vue perçoit l'objet visuel à travers l'air extérieur

comme à travers une partie qui est à la fois conforme à lui [scil. à l'instrument de la vision] et qui lui est attachée, et si la vue seule a reçu cette capacité exceptionnelle, de pair avec la capacité de voir aussi par reflet ( $\dot{\alpha} \nu \alpha-$ $\kappa \lambda \alpha \sigma(\varsigma)$, il est probable que la vue avait besoin de l'arrivée du pneuma lumineux ${ }^{69}$ coulant d'en haut [scil. du cerveau] qui, lorsqu'il tombe sur l'air environnant et, pour ainsi dire, le frappe, le rend semblable à lui-même ${ }^{70}$.

64. PHP 7.4.25 (V, p. $617 \mathrm{~K} .=452$ De Lacy).

65. PHP 7.5.5-7 (V, p. 619 K. = 454 De Lacy). Voir aussi 7.4.24-25 (V, p. 617 K. $=452$ De Lacy).

66. PHP 7.5.31-32 (V, p. $625 \mathrm{~K}$. = p. 460 De Lacy).

67. PHP 7.5.41 (V, p. $627 \mathrm{~K} .=$ p. 460 De Lacy).

68. Ibid. Voir aussi PHP 7.7.20-21 (V, p. $642 \mathrm{~K} .=474$ De Lacy); Alexandre d'Aphrodise (?), De anima libri mantissa, p. 130.17 et 131.25-26 Bruns; Diogène Laèrce, 7.157 (=SVF II, fr. 867); Némésius, De natura hominis, 8 (p. 65.6-8 Morani). Cf. Zahlfleisch 1895, p. 380-382 ; Jaeger 1914, p. 29-30 ; Cherniss 1933; Hahm 1978, p. 66.

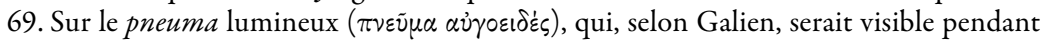
la vivisection des nerfs optiques des grands animaux, voir PHP 7.4.4 (V, p. 609-610 K.

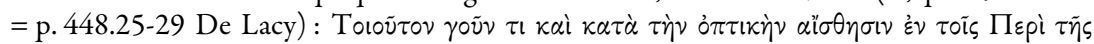

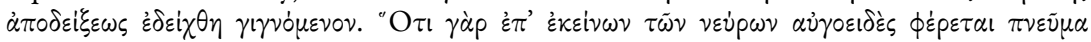

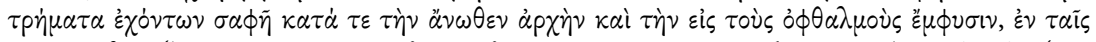

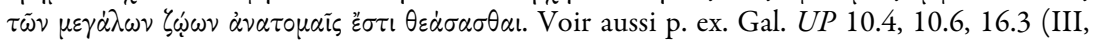




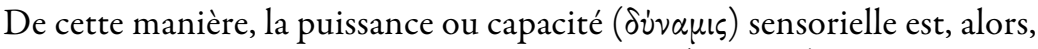
transmise tout du long des ventricules antérieurs (latéraux) de l'encéphale jusqu'à l'interface entre l'air extérieur et l'objet de la vision. Mais qu'est-ce que Galien entend par transmission de puissance ? Sa réponse est explicite, et pourtant non dépourvue de défis interprétatifs : «Ce qui est appelé par la plupart des gens une transmission de puissance est en fait un transfert d'altération qualitative, tel qu'il s'en élève aussi dans l'air par suite de la lumière du soleil ${ }^{71}$. » Une $\delta i \dot{\alpha} \delta \circ \sigma \iota \varsigma \delta v \nu \alpha \dot{\mu} \varepsilon \omega \varsigma$ est par conséquent une $\mu \varepsilon \tau \dot{\alpha}-$

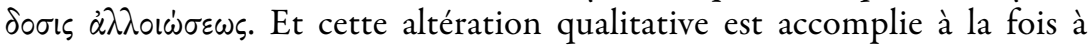
l'intérieur de l'œil, par le pneuma optique sorti du nerf optique, et à l'extérieur de l'œil, c.-à-d. dans le cône visuel d'air extérieur continu, « assimilé » ou «altéré en sa propre nature particulière » par le pneuma optique émis à travers la pupille.

Ici il vaut la peine de rappeler que la principale finalité de la rétine est de percevoir ( $\alpha \dot{i} \sigma \theta \dot{\alpha} \nu \varepsilon \sigma \theta \alpha \iota)$ un changement qualitatif $(\dot{\alpha} \lambda \lambda \circ i \omega \sigma \iota \varsigma)$ dans le cristallin. Il est peu probable que cela soit une référence à l'altération causée par l'arrivée continuelle du pneuma optique dans le cristallin, surtout étant donné que la fonction du flot continu de pneuma optique du cerveau à travers le nerf optique et par l'humeur vitrée jusqu'au cristallin est censément de rendre ce dernier sensible. Si l'altération perçue par la rétine était cette altération, la rétine percevrait la capacité sensible du cristallin. Galien ne suggère toutefois nulle part que la fonction primordiale de la rétine soit la perception de la capacité sensible de l'œil ou la conscience sensible de soi ou n'importe quelle autre version de « l'œil percevant l'œil ». Bien plutôt, une seconde sorte d'altération a lieu dans le cristallin, et c'est elle que perçoit la rétine. En examinant quels genres d'altération qualitative peuvent avoir lieu dans le cône d'air visuel extérieur, on trouve quelque secours pour préciser la nature de ces deux sortes d'altération et du rapport qui les unit.

Non seulement le pneuma optique, quand il sort de l'œil, transmet au cône une altération, en «sensibilisant » l'air qui s'y trouve, mais l'objet visuel perceptible à la base du cône déclenche lui aussi une altération qualitative dans le cône sensibilisé. L'air contenu dans le cône transfère ce dernier changement de la base jusqu'au sommet situé dans la pupille ${ }^{72}$. Il y a donc dans le cône aussi deux sortes d'altération : l'une est provoquée par le pneuma optique, qui sensibilise l'air extérieur au premier contact, et l'autre

p. 780, 785-786 K. ; IV, p. 275 K. = II, p. 70, 74, 384 Helmr.) ; De locis affectis, 4.2 (VIII, p. 218 K.) ; Müller 1895, p. 471-472 ; Mugler 1964, p. 68.

70. PHP 7.5.41 (V, p. $627 \mathrm{~K} .=$ p. 460 De Lacy).

71. PHP 7.4.24 (V, p. $617 \mathrm{~K} .=$ p. 452 De Lacy).

72.PHP 7.7.4 (V, p. $638 \mathrm{~K} .=470$ De Lacy). 
est provoquée dans l'air sensibilisé par l'objet visuel; autrement dit, l'une est déclenchée par le pneuma émanant de l'œil, et l'autre par l'objet vu.

Lorsque le changement qualitatif déclenché par l'objet visuel à la base du cône arrive à la pupille, il est transmis au cristallin ${ }^{73}$. C'est apparemment à cette altération que Galien se réfère quand il affirme que l'utilité primordiale de la rétine est de percevoir les changements qualitatifs dans l'humeur cristalline. Mais s'il en est ainsi, la théorie de Galien comporte des apories supplémentaires. Par exemple, si l'air sensibilisé par le pneuma peut transférer des altérations provoquées par un objet extérieur au cristallin sensibilisé par le pneuma et, à travers le cristallin, à la rétine (qui, comme nous l'avons vu ci-dessus, II, est insérée dans les deux bords latéraux du cristallin), pourquoi le pneuma sensible dans le cristallin ne transmettrait-il pas aussi ces mêmes altérations à l'humeur vitrée sensibilisée par le pneuma ? Et pourquoi la transmission de changement qualitatif ne continuerait-elle pas, à son tour, à remonter de l'humeur vitrée jusqu'au pneuma sensoriel du nerf optique (ou jusqu'à la partie la plus large de la rétine qui couvre l'humeur vitrée), et de là tout du long jusqu'au cerveau ? Ou bien, si le changement qualitatif provoqué par l'objet atteint le cerveau de cette façon, pourquoi Galien s'abstient-il de le dire, et pourquoi rend-il, à la place, les attaches rétiniennes aux bords latéraux du cristallin seules responsables de la perception du changement ? Que la transmission de changement qualitatif par le pneuma optique ne soit pas à sens unique (allant uniquement du cerveau par le nerf optique, etc., vers le cône visuel extérieur) est, après tout, rendu évident par le fait que le pneuma retransmet une altération déclenchée par l'objet visuel jusqu'au cristallin. Ces apories nous ramènent aux problèmes évoqués ci-dessus concernant les rôles respectifs, dans la perception visuelle, de la substance du nerf (c.-à-d. la substance cérébrale, y compris la rétine) et du contenu du nerf (c.-à-d. le pneuma psychique sensoriel qui est, dans ce nerf, le pneuma optique).

73. Voir p. ex. UP 8.6, 10.1, 10.2, 10.15 (III, p. 641, 643-644, 761, 762, 838-841 K. = I, p. 464-465, 466-467 ; II, p. 55-56, 57, 110-113 Helmr.); PHP 7.7.1-4 (V, p. 637-638 K. $=$ p. 470 De Lacy). Si le sommet du cône d'air se trouve à la pupille, l'altération transmise de la base au sommet du cône doit passer à travers la cornée. Comment cela peut se faire, Galien l'explique ainsi : «Dans le cas des yeux, même s'ils sont complètement recouverts de tous les côtés, l'altération venue des couleurs extérieures atteint facilement la partie du cerveau [c.-̀̀-d. la rétine] qui se trouve dans les yeux. Car en réalité, la tunique semblable à de la corne [la cornée] est mince, claire et pure, en sorte qu'elle n'isole totalement ni cette partie, ni l'altération qui chemine à travers elle; et immédiatement après la cornée, à la pupille même, se trouve l'humeur du cristallin, à laquelle est naturellement attachée la partie du cerveau qui se trouve dans les yeux [la rétine]<» (UP 8.6 ; III, p. 643-644 K. = I, p. 466467 Helmr.) 
À l'occasion de la discussion de cette contribution au Centre Léon Robin, il a été suggéré que la réponse pourrait se trouver en partie dans l'emplacement du sommet du cône visuel (fig. b, A). Plus précisément, on a proposé que, puisque la pupille n'est pas un point, mais un petit orifice circulaire, on peut supposer que le sommet du cône s'étend brièvement à travers la pupille jusqu'à un point situé sur le cristallin (c.-à-d. à travers la fig. a, 13, jusqu’à la fig. a, 7). Malgré le silence de Galien là-dessus, c'est une interprétation plausible du sommet, et pourtant il n'est pas évident qu'elle apporte une solution réelle aux énigmes mentionnées ci-dessus. Elle offre néanmoins l'avantage de fournir au cristallin un point de contact physique, substantiel et direct, avec le cône visuel, en rendant ainsi compte du transfert direct du cône au cristallin de l'altération provoquée par l'objet visuel. Mais si la rétine perçoit cette altération, comme l'affirme Galien, alors l'altération doit se répandre à travers tout le cristallin jusqu'à ses bords extérieurs, puisque le principal point de contact de la rétine avec le cristallin se trouve à la plus vaste circonférence de ce dernier (fig. a), bien loin de l'endroit où le sommet du cône rencontrerait le cristallin. Et si l'altération est transmise par le pneuma dans le cristallin tout du long jusqu'aux bords latéraux du cristallin, pourquoi ne serait-elle pas transmise aussi à l'humeur vitrée (d'autant plus que les humeurs cristalline et vitrée sont immédiatement contiguës), puis au nerf optique. etc.? En un mot, un problème fondamental soulevé ci-dessus persiste : les fonctions respectives de la rétine et du pneuma intra-orbitaire dans la réception visuelle et la perception des objets extérieurs. Après tout, comme il a été montré ci-dessus (II), Galien attribue la supériorité cognitive de l'œil sur les autres instruments de perception dans une large mesure à la quantité exceptionnelle de pneuma sensoriel qui coule dans l'œil, et deuxièmement, au fait que l'extrémité rétinienne du nerf optique est elle-même substance cérébrale. En plus, il affirme que le cristallin ne saurait fonctionner comme instrument principal de la vision, si les deux extrémités antérieures de la rétine n'étaient pas insérées dans le cristallin, en sorte que le cerveau, par son extension (le nerf optique et la rétine), puisse percevoir les changements qualitatifs dans le cristallin, qui dérivent en fin de compte des objets de la vision ${ }^{74}$.

On peut aussi chercher quelques lumières en examinant les réponses de Galien à une question supplémentaire: que voyons-nous lorsque nous voyons ? Bien que ses idées présentent quelques lacunes et peut-être incohérences ici aussi, les principales composantes de sa réponse incluent les suivantes. D'abord, il tend à reconnaître avec Aristote et d'autres prédéces-

74. UP 10.2 (III, p. 762, 766-767 K. = II, p. 56-57, 60-61 Helmr.) ; PHP 7.5.26-28 (V, p. $624 \mathrm{~K} .=$ p. 458 De Lacy). 


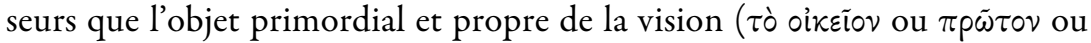
¡ઈı par exemple, Galien explique certaines propriétés de l'œil précisément en termes de fonction de perception de la couleur :

L'instrument de la vue, puisqu'il doit être capable de faire la distinction entre les couleurs, est devenu lumineux, puisque seuls de tels corps sont par nature capables d'être altérés par les couleurs, comme le rend évident l'air environnant, altéré par les couleurs quand il est particulièrement clair ${ }^{76}$.

Dans ce contexte, il vaut la peine de rappeler que Galien décrit à la fois le cristallin et l'humeur vitrée comme étant par nature particulièrement blancs, clairs ou purs ( $\kappa \alpha \theta \alpha \rho o ́ v)$ et brillants $(\lambda \alpha \mu \pi \rho o ́ v)^{77}$, semblables respec-

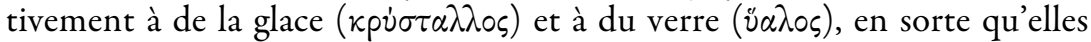
devraient, selon son idée, être exceptionnellement susceptibles d'altérations causées par la couleur, bien que l'humeur vitrée soit inférieure au cristallin pour la limpidité et le brillant.

En second lieu, la couleur, nous dit Galien, crée facilement un changement qualitatif dans l'air. C'est une des raisons pour lesquelles le cône d'air qui se trouve entre l'objet de la vision et l'instrument de la vision peut fonctionner de la manière schématisée ci-dessus : « De la même façon que, sur un simple contact de la lumière du soleil, tout l'air est d'un coup rendu similaire par la lumière du soleil, ainsi, l'air est instantanément transformé par la couleur ${ }^{78}$. » Et ainsi de même, « par nature, l'air brillant, quand il est altéré par la couleur, transmet [envoie au travers] l'altération jusqu'à l'instrument de la vue ${ }^{79}$, c.-à-d. jusqu'au cristallin.

Troisièmement, Galien est toutefois conscient que la perception visuelle inclut beaucoup plus que la discrimination des couleurs. Parmi les objets associés du discernement visuel, il énumère la grandeur ( $\left.\mu \dot{\varepsilon} \gamma \varepsilon \theta_{\circ \varsigma}\right)$, la forme $(\sigma \chi \tilde{\eta} \mu \alpha)$, le mouvement ( $\kappa i \nu \eta \sigma \iota \varsigma)$, la position ( $\theta \dot{\varepsilon} \sigma \iota \varsigma)$, et la distance

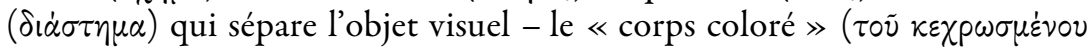
$\sigma \dot{\omega} \mu \alpha \tau \circ \varsigma)$ - du spectateur, soulignant qu'aucun autre organe des sens ne peut les percevoir, si ce n'est le toucher, qui peut percevoir incidemment la

75. Voir Boudon 2002. Galien attribue toutefois à la vue aussi la capacité de percevoir la

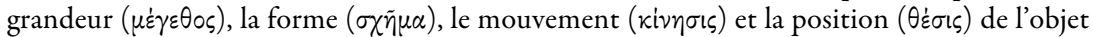
visuel ainsi que sa distance $(\delta \dot{\imath} \dot{\alpha} \tau \eta \mu \alpha)$ du spectateur ; cf. UP 16.3 (IV, p. $273 \mathrm{~K}$; II, p. 383.7 8 Helmr.) ; voir ci-dessous et n. 80-81.

76. PHP 7.7.1 (V, p. $637 \mathrm{~K} .=$ p. 470 De Lacy).

77. P. ex. UP 8.6, 10.1 (III, p. 641, 761 = I, p. 464-465; II, p. 55-56 Helmr.) ; PHP 7.5.26-28 (V, p. 624 K. = p. 458 De Lacy); Anat. adm. 10.3 (p. 47 Simon = p. 39 D. $=$ p. $870-871 \mathrm{G}$.).

78. PHP 7.7.3 (V, p. 638 K. = p. 470 De Lacy).

79. PHP 7.7.4 (V, p. 638 K. = p. 470 De Lacy). 
grandeur et la forme ${ }^{80}$. Il est frappant que tous ces traits des objets perçus par la vue représentent le cœur du programme anatomique de Galien: l'observation de la taille, de la position, de la forme, de l'emplacement et de la couleur des parties du corps est, d'après Galien et ses devanciers hellénistiques, un fondement indispensable de la connaissance anatomique ${ }^{81}$. La ressemblance entre les objets de l'organe visuel et ceux de l'anatomiste fait ainsi de la vue un instrument primordial de l'anatomie, laquelle est à son tour un préalable à la physiologie, à la pathologie et à la thérapeutique.

Quatrièmement, Galien affirme que l'œil voit «le corps qui est vu »

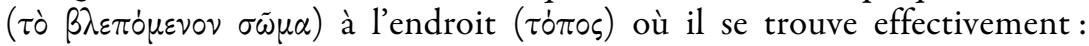
« cela apparaĩt clairement dans la perception même », au point que « les géomètres ne le démontrent même pas mais le posent comme une évidence », bien qu'il y ait des preuves géométriques que nous voyons en lignes droites ${ }^{82}$. L'affirmation que nous voyons les choses «là où elles sont » appelle cependant quelques réserves, comme le montre précisément la démonstration géométrique ( $c f$. ci-dessous IV).

Un examen plus rapproché de «ce que nous voyons » confirme ainsi que la vue est un sens auquel on peut se fier complètement, plus précis qu'aucun autre, et plus apte qu'aucun autre à communiquer à celui qui perçoit des ensembles complexes de données sur les objets extérieurs. Pourtant, les réponses détaillées de Galien à la question « que voyons-nous lorsque nous voyons ?» ne fournissent pas de solution aux apories fondamentales soulevées ci-dessus.

Il nous reste une voie d'exploration significative : le traitement géométrique de la vision par Galien, qu'il doit pour sa plus grande partie à la tradition optique représentée entre autres par Euclide, que Galien désigne par son nom dans ce contexte aussi ${ }^{83}$.

80. PHP 7.5.33-37, 7.6.24, 7.7.20 (V, p. 625-626, 634, 642 K. = p. 460, 466, 474 De Lacy) ; UP 16.3 (IV, p. 273-274 K. = II, p. 383, 4-18 Helmr.). Cf. Némésius, De natura hominis, 7 (p. 59.19-60 Morani).

81. Voir p. ex. Celse, Medicina, 1, prooem. 24 (Corpus medicorum latinorum, I, p. 21 Marx), et Galien, Anat. adm. passim.

82. PHP 7.5.39-40 (III, p. $816 \mathrm{~K} .=$ II, p. $95-96$ Helmr.)

83. UP 10.12-13 (III, p. 812-831 K. = II, p. 92-105 Helmr.). Pour des références à Euclide. voir p. ex. UP 10.13 (III, p. $830 \mathrm{~K}$. = II, p. 105,5-11 Helmr.). Sur les traditions antiques d'optique géométrique, voir Lejeune 1948 ; Lindberg 1976, p. 11-17 ; Smith 1981; Simon 1988. Sur l'optique d'Euclide, voir aussi Pappus, Collection, 6.86-103. Pour l'optique attribuée à Ptolémée, contemporain de Galien, voir Lejeune 1958, 1989 ; Smith 1996. Selon Knorr 1985, l'auteur de l'Optique attribuée à Ptolémée était Sosigénès. 


\section{La géométrie de la vision : lignes, « rayons», et une colonne qui saute}

Parmi les questions suscitées par les adaptations galéniques de la tradition mathématique de l'optique, deux sont particulièrement pertinentes ici. Premièrement, la géométrie de la vision chez Galien fournitelle quelque réponse aux problèmes irrésolus évoqués ci-dessus ? Deuxièmement, cette analyse géométrique se trouve-t-elle dans un rapport cohérent avec ses explications physiologiques et anatomiques de la vue ?

Dans le De usu partium, livre X, Galien présente la géométrie de l'espace visuel comme un trait nécessaire de sa théorie de la vision, mais dont il aurait aimé se passer. Pendant longtemps, dit-il, il a évité de discuter de ce trait, à cause de la résistance de son public aux mathématiques. En effet, il décrit les conséquences sociales des mathématiques comme fort désagréables : si vous vous mettez à parler de mathématiques, les gens commencent à s'ennuyer et par conséquent vous fuient. Un rêve et un daimon ont néanmoins poussé un Galien réticent à passer à la géométrie visuelle ${ }^{84}$.

Comme la tradition d'optique géométrique, Galien soutient l'idée que

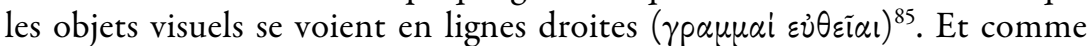
certains de ses précurseurs, il appelle parfois ces lignes ő $\psi \varepsilon ı \varsigma$ ( « actes de vision », « visions »), que les interprètes modernes traduisent normalement par « rayons visuels ${ }^{86}$. Cependant, Galien, à la différence d'Euclide, de Théon d'Alexandrie et d'autres auteurs anciens des traités d'optique

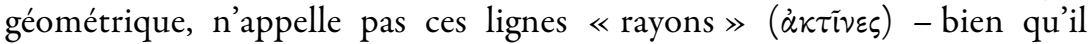
utilise librement le terme de « rayons » pour se référer aux rayons lumineux du soleil ${ }^{87}$ - mais simplement « lignes » $(\gamma p \alpha \mu \mu \alpha i)$ ou « lignes droi-

84. UP 10.12 (III, p. 812,814, 828 K. = II, p. 93.5, 94.9, 103.17 Helmr.) : غ่vن่ $\pi v 10 v$, $\delta \alpha i \mu \omega \nu$. Sur le daimon chez Galien, voir von Staden 2003.

85. UP 10.12 (III, p. 815-818 K. = II, p. 94.22-97.4 Helmr.) ; PHP 7.5.40 (V, p. 627 K. = p. 460 De Lacy). Voir Lejeune 1948, p. 37-41.

86. P. ex. May 1968, p. 471, 493, 495-496; Simon 1988, p. 26-28; Sabra 1989, p. liv, 44; Daremberg, 1854-1856, I, p. 639. Cf. Mugler, 1964, s. v. ö $\psi$ is (et p. 10 sur le « rayon

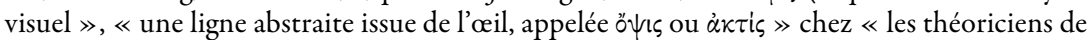
l'optique... à partir d'Euclide »). Mais Siegel 1970a et Lindberg 1976, p. 10-11, suivent les traces de Galien en évitant l'expression « rayon visuel » dans leurs exposés de sa théorie.

87. Pour l'utilisation que fait Galien d' áktis pour faire référence à un rayon de soleil, voir p. ex. UP 10.12 (III, p. $818 \mathrm{~K}$. = II, p. 96,25 Helmr.); De cuiusque animi peccatorum dignotione atque medela libellum, 5. 4-5 (V, p. $82 \mathrm{~K} .=\mathrm{CMG} \mathrm{V,} \mathrm{4,} \mathrm{1,} \mathrm{1,} \mathrm{p.} 55$ DeBoer); De locis affectis 4.8 (VIII, p. 266 K.) ; De tremore, palpitatione convulsione. 6 (VII, p. 621 K.) ; De symptomatum causis, 1.5 (VII, p. $110 \mathrm{~K}$.) ; De compositione medicamentorum per genera, 3.2 (XIII, p. 570 K.) ; De antidotis 1.8 (XIV, p. 47-48 K.) ; In Hippocratis Epidemiarum VI. comm. 6.1.29 (XVIIA, p. $880 \mathrm{~K} .=$ CMG V, 10, 2, 2, p. 49, dans une explication de $\pi \dot{\varepsilon} \mu \phi(\xi)$ ).

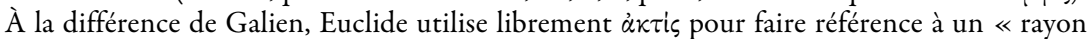
visuel » émanant de l'œil et aux lignes dans le cône visuel (on trouve plus de quatre-vingts 
tes» ou «lignes qui s'étendent comme de fines toiles d'araignée » ou « actes de vision » (ő $\psi \varepsilon ı)$ ). Le fait que Galien évite avec constance le terme traditionnel de « rayons » a apparemment échappé à l'attention de la plupart des érudits modernes, mais il se peut qu'il soit significatif. Il est peutêtre dû, en premier lieu, à la reconnaissance par Galien des limites de l'analogie entre le processus de la vision et les effets du soleil, et deuxièmement, à sa résistance aux versions traditionnelles de la théorie émissionniste de la vision. En particulier, lorsque nous voyons les objets visuels en lignes droites, la pupille, comme il a été montré ci-dessus (III), n'émet son pneuma lumineux qu'à un point situé devant les yeux, mais pas à la distance la plus éloignée (ci-dessus III, note 65). De plus, si nous voyons ce que nous voyons en lignes droites, ce n'est pas parce que l'œil émet des rayons, mais parce que l'air sensibilisé par le pneuma optique dans le cône visuel transfère continûment les altérations ( $\dot{\lambda} \lambda \lambda$ oเ $\omega \varepsilon \varepsilon ı \zeta)$ déclenchées par l'objet visuel en lignes droites de sa base ou cercle (fig. b, BC), c.-à-d. de l'interface de l'air dans le cône avec l'objet, jusqu'au sommet du cône situé à la pupille (fig. b, A). D'après Galien, si un grain de millet est suspendu à l'axe du cône visuel (c.-à-d. à $\mathrm{AD}$ dans la fig. b), il cachera le centre du cercle BC (la base du cône visuel) et empêchera ainsi la pupille de le voir ${ }^{88}$. Galien nous signale ainsi que l'obstruction est due au fait que le millet interrompt le continuum d'air sensibilisé entre l'objet visuel et la pupille, et non à quelque blocage d'un « rayon visuel » émis à partir de l'œil.

Les actes de vision (ö $\psi \varepsilon ı \zeta)$ doivent donc être compris comme une communication ou transmission ( $\mu \varepsilon \tau \dot{\alpha} \delta \circ \sigma \iota \varsigma)$ de changement qualitatif ( $\dot{\alpha} \lambda \lambda \circ \iota^{\prime} \dot{\omega}-$ $\sigma \varepsilon \omega \varsigma)$ le long de lignes droites qui vont de l'objet visuel à la pupille de l'œil. $\grave{A}$ aucun moment ces transferts qualitatifs rectilignes ne se courbent ni ne dévient, pas même lorsqu'ils rencontrent un obstacle à l'intérieur du cône visuel, comme un grain de millet.

Ces principes de base sont amplifiés pour inclure au moins cinq traits supplémentaires de géométrie visuelle, provenant eux aussi en partie de traditions hellénistiques. Premièrement, chaque objet vu n'est pas vu seul ni isolé ; on aperçoit plutôt à la fois aussi d'autres objets autour de lui, parce que l'air sensibilisé par le pneuma optique à l'intérieur du cône visuel peut aussi tomber sur des objets situés au-delà du corps que l'on regarde principalement $^{89}$. Dans la figure $\mathrm{c}$, si A est la pupille et BC la première grandeur

usages d'ảktis de ce type dans l'Optique d'Euclide, et plus de soixante dans l'« édition » de l'Optique d'Euclide faite par Théon d'Alexandrie), alors qu'il n'utilise que onze fois ö $\psi 1 s$ (le plus souvent, comme sujet du verbe $\pi \rho \circ \sigma \pi i \pi \tau \omega$, pour se référer à la vision - ou à un rayon visuel ? - qui « tombe sur » ou « rencontre » un objet visuel).

88. UP 10.12 (III, p. $816 \mathrm{~K} .=$ II, p. $95-96$ Helmr.).

89. UP 10.12 (III, p. 818-821 K. = II, p. 97-98 Helmr.). 
que l'on regarde, $\mathrm{BC}$ sera vue tandis que la grandeur FG sera cachée par BC et par conséquent ne sera pas vue, alors que les grandeurs DF et EG, situées de chaque côté de FG, apparaîtront, bien qu'à une distance différente, de chaque côté de $\mathrm{BC}^{90}$.

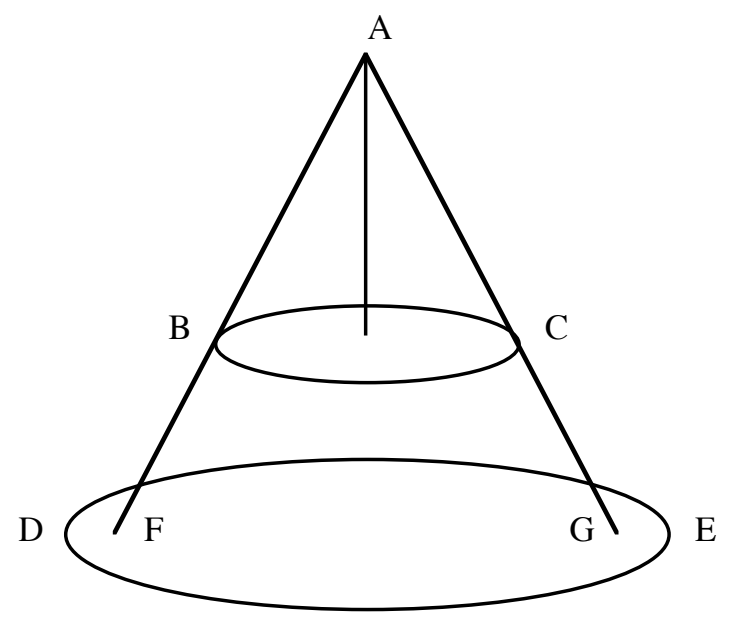

Fig. c

Deuxièmement, l'objet n'est pas vu par l'œil gauche au même endroit que par l'œil droit, ni par les deux yeux au même endroit que par un seul œil. Ainsi, un objet visuel observé seulement par l'œil droit semble se situer plus à gauche lorsqu'il est proche de l'observateur, mais plus à droite lorsqu'il est plus éloigné de lui, alors que, vu par l'œil gauche seulement, le même objet apparaît plus à droite quand il est proche de l'observateur mais plus à gauche quand il est plus éloigné de lui. Mais si le même objet est vu simultanément par les deux yeux, il semble se trouver dans l'espace du milieu, plus au centre ${ }^{91}$. En d'autres termes, comme l'illustre la figure d, relative à d'autres objets visuels, un objet n'est pas vu au même endroit par un oil ou par l'autre ou par les deux yeux à la fois. Dans la figure $\mathrm{d}$, où $\mathrm{A}$ représente la pupille de l'œil droit et $\mathrm{B}$ la pupille gauche, la pupille A seule, sans la coopération de la pupille $\mathrm{B}$, permettra à un observateur de voir la

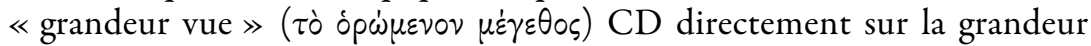
$\mathrm{EF}$, alors que la pupille $\mathrm{B}$ seule permettra de voir la grandeur CD directe- 
ment sur la grandeur GH. Les deux yeux ensemble, à leur tour, verront la grandeur $\mathrm{CD}$ directement sur la grandeur $\mathrm{FG}^{92}$.

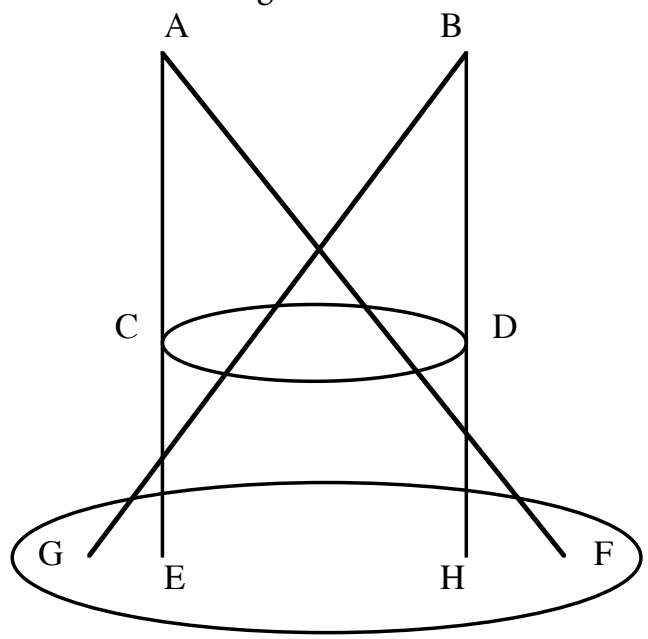

Fig. d

Troisièmement, il est caractéristique des procédés de Galien, tout au long de son analyse de la géométrie de la vision, d'alterner démonstrations

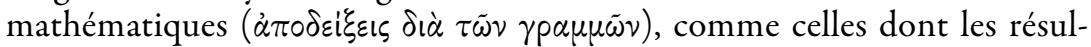
tats sont illustrés par les figures b-d, et expériences $(\pi \varepsilon i \tilde{\rho} \alpha \iota)$ - vivisections comprises - dont le lecteur pourrait lui-même faire l'épreuve ( $\beta \alpha \sigma \alpha \nu i \zeta \varepsilon เ \nu)$ et vérifier ainsi les conclusions de Galien déterminées mathématiquement.

Parmi les tests visuels visant à prouver la validité de la figure $\mathrm{d}$ se trouve, par exemple, « la colonne qui saute » d'un côté à l'autre ( $\dot{\theta} \theta$ póws $\mu \varepsilon \tau \alpha-$ $\pi \eta \delta \tilde{\alpha} \nu)$. Si l'on se tient, affirme Galien, à quelque distance d'une colonne $(\kappa i \omega \nu)$ en la regardant fixement, et si l'on ferme alors alternativement chaque œil, l'un après l'autre, très rapidement, la colonne semblera sauter d'un côté à l'autre. Si l'on ferme l'œil droit, elle semblera se déplacer vers la droite, alors que si l'on ferme l'œil gauche, elle semblera passer de l'autre côté. Et si on la regarde avec les deux yeux en même temps, la colonne semble occuper une place à mi-chemin entre les places où elle apparaît pour chaque œil séparément ${ }^{93}$. « La colonne qui saute » prouve ainsi par un test $(\delta i \dot{\alpha} \tau \tilde{\eta} s \pi \varepsilon i \rho \alpha \varsigma)$ ce que la figure d prouve «par lignes » $(\delta i \dot{\alpha} \tau \tilde{\omega} \nu \gamma \rho \alpha \mu \mu \tilde{\omega} \nu)$ 
ou mathématiquement ${ }^{94}$. Galien se sert encore d'autres doubles procédures de ce genre - alternant démonstrations géométriques et expériences ou tests d'observation - pour asseoir l'autorité d'autres traits de sa théorie de la vision, par exemple, de sa théorie des causes de la vision double (cf. cidessous). En outre, de temps en temps il utilise aussi une alternance entre des explications géométriques et non géométriques sans avoir recours à des expériences ou à des tests.

Quatrièmement, il arrive parfois que cette coexistence entre raisonnement mathématique et non mathématique ne soit pas pacifique à tous égards. Elle mène non seulement à des explications divergentes du même phénomène, mais aussi à des tensions dans la théorie de Galien. Dans le cas du chiasma optique, par exemple, la raison géométrique avancée pour l'existence de cette structure anatomique unique est qu'elle assure la présence des pupilles des deux yeux, droite et gauche, sur le même plan géométrique et prévient ainsi la double vision ${ }^{95}$. Cependant, l'explication physiologique proposée par Galien de la finalité du chiasma optique est la suivante : au cas où l'un des yeux serait ou fermé ou complètement estropié, le pneuma optique, dérivant des deux ventricules antérieurs du cerveau par les deux nerfs optiques, se rendra, grâce au chiasma optique, tout entier dans l'autre œil, qui gagnera ainsi une plus grande puissance ( $\delta \dot{v} v \alpha \mu \varsigma)$ visuelle que d'habitude pour compenser la perte d'un œil ${ }^{96}$. Dans cet exemple, l'explication physiologique du telos du chiasma optique ne fait pas référence à l'explication géométrique de son telos, ni l'explication géométrique à l'explication physiologique. Chacune des deux explications est plutôt présentée comme une explication téléologique, autonome et suffisante du chiasma optique. Il est bien connu que Galien affirme souvent qu'il y a de multiples explications téléologiques d'un seul et même phénomène, et ici aussi il essaie de devancer des critiques de ses procédures explicatives par la remarque suivante : «La nature crée certaines choses dans un but principal

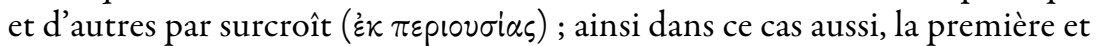
la plus nécessaire utilité [du chiasma optique], c'est que chacun des objets

94. UP 10.12 (III, p. $822 \mathrm{~K} .=$ II, p. 99. 16-18 Helmr.) : « Mais si quelqu'un ne comprenait pas ces démonstrations par le biais des lignes ( $\tau \alpha i \check{\zeta} \delta i \dot{\alpha} \tau \tilde{\omega} \nu \gamma p \alpha \mu \mu \tilde{\omega} \nu \dot{\alpha} \pi \circ \delta \varepsilon i \xi \xi \sigma \sigma \nu)$, il peut se convaincre nettement, s'il a vérifié $(\beta \alpha \sigma \alpha \nu i \sigma \alpha \varsigma)$ le raisonnement par une expérience

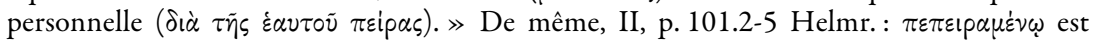
opposé à $\delta$ ì̀ $\tau \tilde{\omega} \nu \gamma p \alpha \mu \mu \tilde{\omega} \nu$.

95. UP 10.13 (III, p. 828-830 K. = II, p. 103-105 Helmr.).

96. UP 10.14 (III, p. 835-837 K. = II, p. 109-110 Helmr.). Sur le chiasma optique voir aussi n. 12-13 supra. 
visuels extérieurs ne soit pas vu double, tandis que l'utilité [physiologique] dont il s'agit maintenant est la seconde ${ }^{97}$. »

Cinquièmement, des exposés de finalité divergents apparaissent aussi lorsque Galien reste dans le domaine de l'explication géométrique. Ses exposés des raisons de la sphéricité imparfaite du cristallin - « l'instrument principal de la vision $\gg-$ en fournit un exemple. Une première explication, fondée sur la nature de l'intersection de différentes sortes de sphères, est que la finalité de la forme elliptique, relativement plate, du cristallin est de lui assurer de la stabilité. En effet, explique Galien, un objet inséré ou reposant sur un corps parfaitement sphérique est plus mobile que sur une surface plus plane, étant porté sur une surface convexe et pour cette raison glissante. « C'est la cause ( $\alpha i \tau i \alpha)$ même de la forme du cristallin », car avec cette forme le cristallin peut reposer avec plus de stabilité sur la surface antérieure de l'humeur vitrée (qui est elle aussi un corps sphérique : fig. a, 6), qu'il ne l'aurait fait s'il avait été parfaitement sphérique ${ }^{98}$.

Par la suite, Galien propose cependant une explication géométrique : la finalité de la forme plus elliptique ou plate du cristallin est de lui permettre de « voir » davantage.

Les figures e et f illustrent une partie de cette démonstration. Dans la figure e, si $\mathrm{AB}$ représente le diamètre de la pupille, cercle parfait, et $\mathrm{CD}$ celui d'un cristallin imaginaire, parfaitement sphérique, EF serait alors la partie du cristallin qui communique avec les objets perçus. Dans la figure $f$, par contraste, les conséquences avantageuses d'un cristallin moins convexe

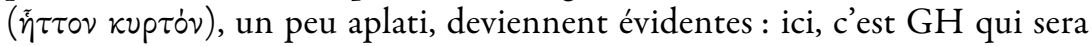

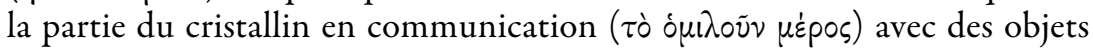
perceptibles. Ici, Galien répète qu'un corps parfaitement sphérique communiquera par moins de ses parties avec les objets vus, mais un corps plus plan, comme le cristallin, par plus de ses parties. GH dans la figure f sera donc plus grand qu'EF dans la figure $\mathrm{e}^{99}$.

97. UP 10.14 (III, p. 837 K. = II, p. 110, 3-7 Helmr.). Pourtant, dans le cas présent il ne s'agit pas de différentes choses - les unes « créées par la nature dans un but principal, les autres par surcroît »- mais plutôt d'une seule et même chose créée par la nature : le chiasma optique, pour lequel Galien apporte des finalités divergentes (voir infra n. 104). Ailleurs

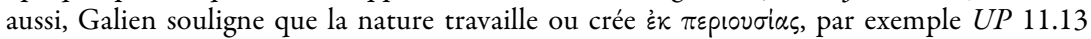

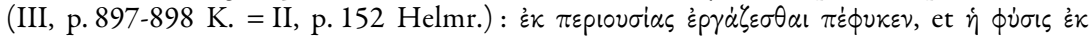

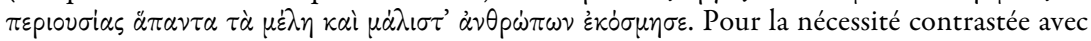
ce qui est « de la profusion » ou « du superflu » ou «du surplus » dans d'autres contextes, voir Aristote, Top. III, 2, 118a5-15 ; Pol. VII, 10, 1329b25-30 ; cf. Démocrite, fr. 144 D.-K.

98. UP 10.6 (III, p. $788-789 \mathrm{~K} .=$ II, p. 76 Helmr.).

99. UP 10.15 (III, p. 838-841 K. = II, p. 111-113 Helmr.). 


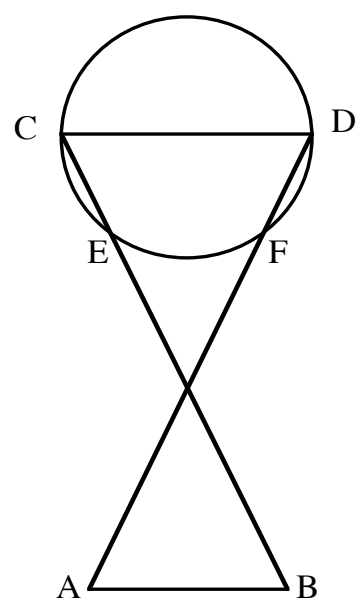

Fig. e

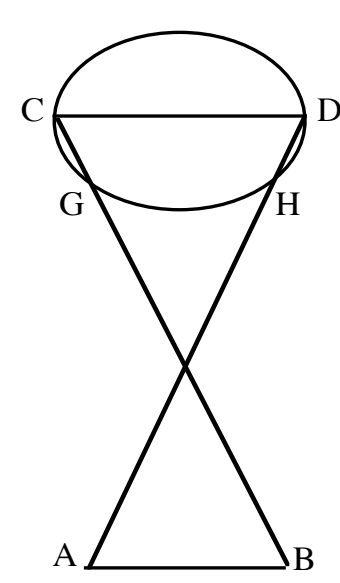

Fig. $f$

Cette dernière explication géométrique de la finalité du cristallin implique, comme Galien l'affirme, que si le cristallin était parfaitement plat, c'est-à-dire s'il était exactement un plan ( $\dot{\pi i} i \pi \varepsilon \delta \circ \nu)$, il «verrait » encore davantage, parce qu'il communiquerait tout entier, dans son ensemble - et pas seulement avec une partie de sa surface antérieure - avec les objets perçus. Pourquoi donc alors la nature, suprême démiurge dont chaque action et création, d'après Galien, est explicable téléologiquement, n'a-t-elle pas fait le cristallin parfaitement plat au lieu d'aplati seulement au point d'être elliptique ? Ici, Galien se sert d'une raison non mathématique pour expliquer cette limitation mathématico-morphologique imposée à notre champ de vision : le cristallin doit en fait être arrondi ( $\pi \varepsilon p$ ı $\phi p \eta \dot{\zeta})$ et elliptique pour résister aux lésions (pour être $\delta v \sigma \pi \alpha \theta \eta \dot{\eta})^{100}$. La forme du cristallin n'est donc pas une imperfection limitatrice mais une autre « merveilleuse œuvre

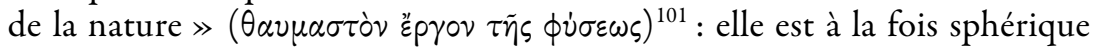
(et donc résistante aux lésions) et aplatie (et donc susceptible de communiquer par la plupart de ses parties avec les objets sensibles).

Ces explications téléologiques multiples d'un seul et même phénomène ne sont pas forcément incompatibles ${ }^{102}$, mais Galien a tendance à présenter chacune comme une explication adéquate et autonome. De plus, il n'est pas évident si et comment Galien concilie ses conclusions sur la finalité d'une 
plus large surface GH (figure f) et sa théorie qu'une pupille élargie entraîne une vision appauvrie, ou, en ce qui concerne notre problème, sa théorie que seul le sommet du cône extérieur visuel d'air sensibilisé rencontre l'œil à la pupille. Après tout, le sommet touche l'œil - ou peut-être le cristallin, comme il a été suggéré ci-dessus (III) - en un seul point, et cela mène à poser la question de la nature du rapport entre ce point A dans la fig. b, et l'efficacité vantée de GF dans la fig. f. Cette question aussi, Galien la passe sous silence. De surcroît, les preuves géométriques citées ci-dessus ne font nulle part référence à la perception rétinienne $(\alpha \grave{l} \sigma \theta \eta \sigma \varsigma)$ des changements dans le cristallin et n'en rendent pas compte, bien que ce soit cette perception rétinienne qui soit la vision selon Galien (comme on l'a montré en III). La preuve illustrée par les figures e et $\mathrm{f}$ ne situe donc pas le sommet du cône visuel sur le cristallin, pas plus qu'elle ne démontre pourquoi la rétine, qui est en contact direct avec le cristallin à ses extrémités périphériques les plus éloignées ( $c f$. ci-dessus II, fig. a, 7 et 5 ), tirerait plus de profit d'un cristallin elliptique, si, comme on l'a montré plus haut (III), le pneuma sensoriel est diffusé de manière égale dans tout le cristallin.

Galien réussit fréquemment à établir un rapport cohérent entre ses explications géométrique, physiologique et anatomique de la vue et de ses instruments, soit en arrivant au même point doctrinal par chacune de ces approches, soit en utilisant les différentes explications pour établir de multiples finalités complémentaires et non contradictoires. Parfois, cependant, les fils anatomiques, physiologiques et géométriques entrent en rivalité sans résolution, ou du moins, se retrouvent dans une relation de juxtaposition ou surimposition qui crée des tensions et même des apories à l'intérieur de son système remarquablement détaillé. L'observation anatomique lui enseigne, par exemple, que la rétine - extension de la substance du nerf optique et par là de celle du cerveau - doit jouer un rôle central dans la perception visuelle, alors que la physiologie impose à son système le pneuma psychique sensoriel lumineux que la rétine peut contenir mais non diriger, et dont sa géométrie de la vision ne semble savoir que faire (au moins de celui contenu dans les deux corps sphériques sensibles du globe oculaire).

Malgré tout le brillant des observations de Galien sur l'œil et le cerveau, et malgré toute la richesse de ses explications détaillées, à multiples facettes, de nombreux aspects de la vision, sa théorie de la vision, extrêmement influente, présente plusieurs apories irrésolues et plusieurs lacunes. Sa rhétorique de cohérence systématique ainsi que la facilité et l'agilité extraordinaires avec lesquelles il a recours à des modes d'explication divergents voilent parfois les lacunes et les tensions au sein de sa théorie. De temps en temps, cependant, il signale que les difficultés n'ont pas échappé complètement à son attention : «Peut-être quelqu'un m'interrompra-t-il pour me demander comment, si j'ai omis volontairement beaucoup de choses, cet exposé 
[de l'œil et de la vision] est complet... À cela la réponse est prompte... : puisque notre démiurge est tellement habile que chacune des ses œuvres n'a pas une seule utilité mais deux, trois et fréquemment un plus grand nombre, par cela même il est des plus facile d'en omettre quelques-unes des plus obscures pour les gens ordinaires ${ }^{103}$. $\gg$

103. UP 10.15 (III, p. 838 K. = II, p. 110-111 Helmr.). 


\section{BIBLIOGRAPHIE}

BARNES, J. 1982: «Medicine, experience and logic», dans J. Barnes, J. Brunschwig, M. Burnyeat \& M. Schofield (éd.), Science and Speculation: studies in Hellenistic theory and practice, Cambridge-Paris, 1982, p. 24-68.

- 1991 : «Galen on logic and therapy », dans F. Kudlien \& R. J. Durling (éd.), Galen's Method of Healing: Proceedings of the 1982 Galen Symposium, Leyde, 1991 (Studies in Ancient Medicine, 1), p. 50-102.

- 1993: «Galen and the utility of logic », dans J. Kollesch \& D. Nickel, Galen und das hellenistische Erbe: Verhandlungen des IV. internationalen Galen-Symposiums veranstaltet vom Institut für Geschichte der Medizin am Bereich Medizin (Charité) der Humboldt-Universität zu Berlin, 18.-20. September 1989, Stuttgart, 1993, p. 3352.

- 2003: « Proofs and syllogisms in Galen », dans Barnes \& Jouanna (éd.) 2003, p. 1-29.

BARNES, J. \& J. JouAnNa (éd.) 2003 : Galien et la philosophie, Vandœuvres-Genève, 2003 (Entretiens sur l'Antiquité classique, 49).

BOUDON, V. 2002 : « La théorie galénique de la vision : couleurs du corps et couleurs des humeurs », dans L. Villard (éd.), Couleurs et vision dans l'Antiquité classique, Rouen, 2002, p. 65-75.

- 2003: «Art, science et conjecture chez Galien », dans Barnes \& Jouanna (éd.) 2003, p. 269-305.

Cambiano, G. 2000 : « Galeno, Erasistrato e la teleologia di Aristotele », dans D. Manetti (éd.), Studi su Galeno: scienza, filosofia, retorica e filologia. Atti del seminario, Firenze 13 novembre 1998, Florence, 2000 (Studi e testi. Università di Firenze, Dipartimento di Scienze dell'Antichità, 17), p. 45-57.

Chiaradonna, R. 2009 : « Le traité de Galien Sur la démonstration et sa postérité tardoantique », dans R. Chiaradonna \& F. Trabattoni (éd.), Physics and philosophy of nature in Greek Neoplatonism: proceedings of the European Science Foundation Exploratory Workshop (Il Ciocco, Castelvecchio Pascoli, June 22-24, 2006), LeydeBoston, 2009, p. 43-77.

- 2011: «The universal generalization problem and the epistemic status of ancient medicine : Aristotle and Galen », dans C. Cellucci, E. Grosholz \& E. Ippoliti (éd.), Logic and Knowledge, Cambridge, 2011, p. 151-167.

CHERNISS, H. 1933: « Galen and Posidonius' theory of vision » arranged from notes left by the late Professor Roger M. Jones, American Journal of Philology, 54 (1933), p. 154161; repris dans Selected Papers (L. Tarán éd.), Leyde, 1977, p. 447-454.

DAREMBERG, Ch. 1854-1856: CEuvres anatomiques, physiologiques et médicales de Galien, 2 vol., Paris, 1854-1856.

DeBRU, A. 1996: Le corps respirant: la pensée physiologique chez Galien, Leyde, 1996 (Studies in Ancient Medicine, 13).

DONINI, P. 2008 : « Psychology », dans Hankinson (éd.) 2008b, p. 184-209.

DuCKWORTH, W. L. H. (trad.), M. C. Lyons \& B. Towers (éd.) 1962: Galen. On Anatomical Procedures: The Later Books, Cambridge, 1962.

EASTWOOD, B. S. 1980 : «Cosmology and Physiology in Galenic visual theory: Hunayn Ibn Ishāq », Transactions and Studies of the College of Physicians of Philadelphia, 2 (1980), p. 276-283.

- 1982: The elements of vision: the micro-cosmology of Galenic visual theory according to Hunayn Ibn Ishäq, Philadelphie, 1982 (Transactions of the American Philosophical Society, t. $72, n^{\circ} 5$ ). 
FrEDE, M. 1981 : «On Galen's epistemology », dans Nutton 1981, p. 65-86 (repris dans M. Frede, Essays in Ancient philosophy, Minneapolis, 1987, p. 279-298).

GAROFALO, I. (éd.) 1988 : Erasistrati fragmenta, Pise, 1988.

- (trad.) 1991a : Galeno: Procedimenti anatomici, introduzione, traduzione e note, 3 vol., Milan, 1991 (I classici della BUR).

- 1991b : « The six classes of animals in Galen », dans López Férez (éd.) 1991, p. 73-87.

HAHM, D. E. 1978: «Early Hellenistic theories of vision and the perception of color », dans Machamer \& Turnbull (éd.) 1978, p. 60-95.

HANKINSON, R. J. 1991a: « Galen on the foundations of science », dans López Férez (éd.) 1991, p. 15-29.

- 1991b : «Greek medical models of mind », dans S. Everson (éd.), Psychology, Cambridge, 1991 (Companions to Ancient Thought), p. 194-217.

— 1991c: « Galen's anatomy of the soul », Phronesis, 36 (1991), p. 197-233.

- 1993: «Actions and passions: affection, emotion and moral self-management in Galen's philosophical psychology », dans J. Brunschwig \& M. C. Nussbaum (éd.), Passions and Perceptions: Studies in Hellenistic philosophy of Mind. Proceedings of the Fifth Symposium Hellenisticum, Cambridge-New York, 1993, p. 184-222.

- 1998: Galen On Antecedent Causes. Edited with an introduction, translation and commentary, Cambridge-New York, 1998 (Cambridge classical texts and commentaries, 35).

- 2006: « Body and Soul in Galen », dans R. A. H. King (éd.), Common to Body and Soul: Philosophical Approaches to Explaining Living Behaviour in Greco-Roman Antiquity, Berlin, 2006, p. 232-258.

- 2008a : «Epistemology », dans Hankinson (éd.), 2008b, p. 157-183.

- (éd.) 2008b : The Cambridge Companion to Galen, Cambridge (Cambridge Companions), 2008.

HARRIS, C. R. S. 1973: The Heart and the Vascular System in Ancient Greek Medicine from Alcmaeon to Galen, Oxford, 1973.

Havrda, M. 2011 : « Galenus Christianus ? The Doctrine of Demonstration in Stromata VIII and the Question of its Source », Vigiliae Christianae, 65, 2011, p. 343-375.

Helmreich, G. (éd.) 1907 : Galeni de usu partium libri XVII... rec. —, Leipzig 1907 (Bibliotheca script. graec. et roman. Teubneriana).

JAEger, W. 1914: Nemesios von Emesa: Quellenforschungen zum Neuplatonismus und seinen Anfängen bei Poseidonios, Berlin, 1914.

KNORR, W. 1985: «Archimedes and the Pseudo-Euclidean Catoptrics: Early Stages in the Ancient Geometric Theory of Mirrors », Archives internationales d'histoire des sciences, 35 (1985), p. 28-105.

KüHN, K. G. 1821-1833: Claudii Galeni Opera omnia ed. cur. —, Leipzig, 1821-1833 (= Medicorum Graecorum quae exstant, vol. 1-20). [Réimpr. Hildesheim, 19861997.]

LeHOuX, D. 2007 : « Observers, Objects, and the Embedded Eye ; or Seeing and Knowing in Ptolemy and Galen », Isis, 98 (2007), p. 447-467.

Lejeune, A. 1948 : Euclide et Ptolémée: deux stades de l'optique géométrique grecque, Louvain, 1948 (Université de Louvain, Recueil de Travaux d'Histoire \& de Philologie, $3^{\text {e }}$ Sér., fasc. 31 ).

- 1958 : «Les recherches de Ptolémée sur la vision binoculaire », Janus, 47 (1958), p. 79 86.

- 1989: L'Optique de Claude Ptolémée dans la version latine d'après l'arabe de l'émir Eugène de Sicile, deuxième édition critique et exégétique augmentée d'une traduction française et de compléments, Leyde, 1989 (Collection de travaux de l'Académie 
internationale d'histoire des sciences, 31) [ $1^{\mathrm{re}}$ éd. Louvain, 1956 (Université de Louvain, Recueil de Trav. d'Hist. \& de Philol., $4^{\mathrm{c}}$ Série, fasc. 8)].

LiNDBERG, D. C. 1967 : «Alhazen's Theory of Vision and its Reception in the West », Isis, 58 (1967), p. 321-341.

- 1976: Theories of Vision from al-Kindi to Kepler, Chicago, 1976.

- 1978: « The intromission-extramission controversy in Islamic visual theory: Alkindi versus Avicenna », dans Machamer \& Turnbull (éd.) 1978, p. 137-159.

LLOYD, G. E. R. 1983 : Science, Folklore and Ideology : Studies in the Life Sciences in Ancient Greece, Cambridge, 1983.

LÓPEZ FÉreZ, J. A. (éd.) 1991: Galeno: obra, pensamiento e influencia (coloquio internacional celebrado en Madrid, 22-25 de Marzo de 1988), Madrid, 1991.

Machamer, P. K. \& R. G. Turnbull (éd.) 1978 : Studies in Perception: Interrelations in the History of Philosophy and Science, Columbus, Ohio, 1978.

MANZONI, T. 2001 : Il cervello secondo Galeno (Collana di Scienze Umane, Università degli Studi di Ancona Facoltà di medicina e chirurgia), Ancona, 2001.

Manuli, P. \& M. Vegetti (éd.) 1988: Le opere psichologiche di Galeno: Atti del terzo colloquio galenico internazionale, Pavia, 10-12 settembre 1986, Naples, 1988 (Elenchos, 13).

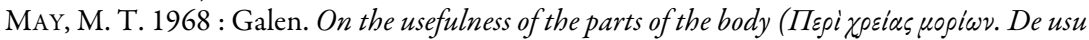
partium), translated with introduction \& commentary, 2 vol., Ithaca, New York, 1968.

Moraux, P. 1984 : Der Aristotelismus bei den Griechen von Andronikos bis Alexander von Aphrodisias, vol. 2 : Der Aristotelismus im I. und II. Jh. n. Chr., Berlin-New York, 1984 (Peripatoi, 6).

MORISON, B. 2008: « Logic », dans Hankinson (éd.) 2008b, p. 66-115.

MUGLER, CH. 1964: Dictionnaire historique de la terminologie optique des Grecs: douze siècles de dialogues avec la lumière. Paris, 1964 (Études \& Commentaires, 53).

MÜLLER, I. von 1895: «Über Galens Werk vom wissenschaftlichen Beweis », Abhandlungen der königlichen bayerischen Akademie der Wissenschaften, 20 (1895), p. 403478.

NutTon, V. (éd.) 1981 : Galen: Problems and Prospects. A collection of papers submitted at the 1979 Cambridge Conference, Londres, 1981.

- (éd.) 2002: The Unknown Galen, Londres, 2002 (Bulletin of the Institute of Classical Studies, Supplement 77).

PINES, S. 1955 : « Rāzī critique de Galien », Actes du VII Congrès International d'Histoire des Sciences (Jerusalem, 4-12 août 1953), Paris, 1955 (Collection des Travaux de l'Académie internationale d'Histoire des Sciences, 8), p. 480-487; repris dans S. Pines, Studies in Arabic versions of Greek texts and in mediaeval science, Jérusalem, 1986 (The collected works of S. Pines, vol. 2), p. 256-263.

ReINHARDT, K. 1926: Kosmos und Sympathie: neue Untersuchungen über Poseidonios, Munich, 1926.

RocCA, J. 2002: « The brain beyond Kühn : reflections on Anatomical Procedures, Book IX », dans Nutton (éd.) 2002, p. 87-100.

- 2003: Galen on the Brain: Anatomical Knowledge and Physiological Speculation in the Second Century AD, Leyde-Boston, 2003 (Studies in Ancient Medicine, 26).

Roselli, A. 1999: « Notes on the doxai of doctors in Galen's commentaries on Hippocrates », dans van der Eijk (éd.) 1999, p. 359-381.

SABRA A. I. 1978 : « Sensation and inference in Alhazen's theory of visual perception », dans Machamer \& Turnbull (éd.) 1978, p. 160-185 (repris dans A. I. Sabra, Optics, Astronomy, and Logic: Studies in Arabic science and philosophy, Aldershot, 1994 (Variorum collected studies series, 444), $\mathrm{n}^{\circ} \mathrm{X}$ ). 
- 1989: The optics of Ibn al-Haytham, Books I-III: on direct vision, 2 vol., Londres, 1989.

SAVAGE-Smith, E. 2002 : «Galen's lost ophthalmology and the Summaria Alexandrinorum », dans Nutton (éd.) 2002, p. 121-138.

SIEGEL, R.E. 1970a : «Principles and contradictions of Galen's doctrine of vision », Sudhoffs Archiv, 54 (1970), p. 261-276.

- 1970b: Galen on sense perception: his doctrines, observations and experiments on vision, hearing, smell, taste, touch and pain, and their historical sources, Bâle-New York, 1970. [= Galen's system of physiology and medicine, II.]

SimON, G. 1988 : Le regard, l'être et l'apparence dans l'optique de l'antiquité, Paris, 1988 (Des Travaux).

Simon, M. (éd., trad., comment.) 1906: Sieben Bücher Anatomie des Galen: ANATOMIKSN EIXEIPH $\Sigma E \Omega N ~ B I B A I \Omega N ~ \Theta-I E$, zum ersten Male veröffentlicht nach den Handscriften einer arabischen Übersetzung des 9. Jahrh. n. Chr. ins Deutsche übertragen und kommentiert, 2 vol., Leipzig, 1906. [Réimpr. Francfort-surle-Main, 1996, (Publications of the Institute for the History of Arabic-Islamic Science, Islamic Medicine, vol. 16-17).]

SMITH, A. M. 1981 : « Saving the appearances of the appearances : the foundation of classical geometric optics », Archive for history of the exact sciences 24.2 (1981), p. 73-99.

- 1996: Ptolemy's theory of visual perception: An English translation of the Optics with introduction and commentary, Philadelphie, 1996 (Transactions of the American Philosophical Society, 86, 2).

Staden, H. von 1989 : Herophilus. The Art of Medicine in Early Alexandria, edition, translation and essays, Cambridge, 1989 (réimpr. 1994).

- 1991: «Galen as historian : his use of sources on the Herophileans », dans López Férez (éd.) 1991, p. 205-222.

- 2000 : «Body, soul, and nerves. Epicurus, Herophilus, Erasistratus, the Stoics, and Galen », dans J. P. Wright \& P. Potter (éd.), Psyche and soma. Physicians and metaphysicians on the mind-body problem from Antiquity to the Enlightenment, Oxford, 2000, p. 79-116.

- 2002 : «L'œil d'après Hérophile, Démosthène Philaléthès et Aglaïas de Byzance », dans Autour de l'ail dans l'antiquité. Approche pluridisciplinaire. Table ronde de Lons-leSaunier-Jura - 11-12 février 1994, Lons-le-Saunier, 2002, p. 83-93.

- 2003: «Galen's daimon: reflections on 'irrational' and 'rational' », dans N. Palmieri (éd.), Rationnel et irrationnel dans la médecine ancienne et médiévale: aspects historiques, scientifiques et culturels (Centre Jean Palerne, Mémoires, 26), SaintÉtienne, 2003, p. 15-43.

- 2012: «The Physiology and Therapy of Anger: Galen on Medicine, The Soul, and Nature », dans F. Opwis \& D. Reisman (éd.), Islamic Philosophy, Science, Culture, and Religion: Studies in Honor of Dimitri Gutas, Leyde-Boston, 2012, p. 63-87 (Islamic Philosophy, Theology and Science, 93).

Tieleman, T. 1996: Galen and Chrysippus on the Soul: Arguments and refutations in the 'De Placitis' Books II-III, Leyde, 1996 (Philosophia Antiqua, 68).

- 2003: « Galen's psychology », dans Barnes \& Jouanna 2003, p. 131-169.

- 2008: « Methodology », dans Hankinson (éd.) 2008b, p. 49-65.

VAN DER EIJK, Ph. J. (éd.) 1999 : Ancient histories of medicine: essays in medical doxography and historiography in classical antiquity, Leyde, 1999 (Studies in Ancient Medicine, 20).

- 2010: «Galens Auseinandersetzung mit Aristoteles' Ansichten zum Gesichts- und Geruchssinn », Antike Naturwissenschaft und ihre Rezeption, 20 (2010), p. 81-107.

VegETTI, M. 1981 : « Modelli di medicina in Galeno », dans Nutton (éd.) 1981, p. 47-63. 
— 1994: «L'immagine del medico e lo statuto epistemologico della medicina in Galeno », dans Aufstieg und Niedergang der römischen Welt, II.37.2, Berlin-NewYork, 1994, p. 1672-1717.

- 1999: « Historiographical strategies in Galen's physiology (De usu partium, De naturalibusfacultatibus) », dans van der Eijk (éd.) 1999, p. 383-395.

ZahlfleisCH, J. 1895: «Die Polemik des Alexander von Aphrodisias gegen die verschiedenen Theorien des Sehens », Archiv für Geschichte der Philosophie, 8 (1895), p. 373-386. 